\title{
Electromechanical finite element modelling for dynamic analysis of a cantilevered piezoelectric energy harvester with tip mass offset under base excitations
}

\author{
M F Lumentut and I M Howard \\ Laboratory for Dynamic Systems of Smart Structure and Vibration, \\ Theoretical and Applied Mechanics, Department of Mechanical Engineering, \\ Curtin University, Australia
}

\begin{abstract}
A new electromechanical finite element modelling of a vibration power harvester and its validation with experimental studies are presented. The new contributions for modelling of the electromechanical finite element piezoelectric unimorph beam with tip mass offset under base excitation encompass five major solution techniques. These include the electromechanical discretisation, kinematic equations, coupled field equations, Lagrangian electromechanical dynamic equations and orthonormalised global matrix and scalar forms of electromechanical finite element dynamic equations. Such techniques have not been rigorously modelled previously from other researchers. There are also benefits in presenting the proposed numerical techniques. First, the proposed numerical techniques can be used for applications in many different geometrical models including MEMS power harvesting devices. Second, applying tip mass offset located after the end of the piezoelectric beam length can give a very practical design in order to avoid the direct contact of piezoelectric material because of its brittle nature. Since the surfaces of actual piezoelectric material are covered evenly with thin conducting electrodes for generating the single voltage, the new electromechanical discretisation consisting of the mechanical and electrical discretised elements is introduced. Moreover, the reduced electromechanical finite element dynamic equations can be further formulated to obtain the series form of new multimode electromechanical frequency response functions (FRFs) of the displacement, velocity, voltage, current and power including optimal power harvesting. The normalised numerical strain node and eigenmode shapes are also further formulated using numerical discretisation. Finally, the parametric numerical case studies of the piezoelectric unimorph beam under resistive shunt circuit show good agreement with the experimental studies.
\end{abstract}

Keyword: electromechanical discretisation, finite element, MEMS, piezoelectric, power harvesting, smart sensor, strain node, vibration. 


\section{Introduction}

Recently, piezoelectric power harvesting device has shown the viability for converting mechanical energy into useful electrical energy that can be used to power remote smart wireless sensor systems. The applications include the monitoring of intelligent infrastructure, aircraft and environmental monitoring systems and biosensors for the human body. As a profound alternative energy source, a few published literature reviews [1]-[7] have discussed the piezoelectric vibration-based power harvester applications from macro-to-micro levels as giving the benefit to researchers for investigating further novel techniques. To design self-charging and lifespan energy harvesting devices, it requires the technical knowledge for understanding the mechanical and electrical tuning techniques, material or physical properties, mechanical energy sources, power conditioning electronic circuits, sensor systems and miniature fabrication. However, before conducting a manufacturing process for these smart devices in such conditions, initial mathematical models using analytical and numerical techniques become an important aspect for analysing the electromechanical piezoelectric vibration power harvesting response behaviours. Consequently, the development of new concepts has spurred the attention from some researchers. Starting with the simplified analytical lumped parameter models of piezoelectric structures [8]-[10], the electrical equivalent systems representing the electromechanical piezoelectric component and standard circuit interface were developed. Considering the required accuracy, and the necessity to model the cantilevered piezoelectric power harvester with tip mass under base excitation, the development of analytical approaches using Rayleigh-Ritz methods were explored to analyse various parametric case studies for vibration power harvesters [11]-[13]. Kim et al [14] investigated the vibrational piezoelectric bimorph power harvester with the effects of tip mass offset using the Rayleigh-Ritz's method. Their interest and focus were to model the system responses of short and open circuit resonance frequency with variable load resistances using tip mass configuration. Moreover, Erturk [15] further developed comprehensive analytical approaches using assumed-mode methods for Euler-Bernoulli, Rayleigh, and Timoshenko piezoelectric beams with axial deformations. It was proved that by increasing certain number of modes in the choice of admissible trial functions, the approximation solution converges to the analytical solution, especially for the fundamental resonance frequency response. Research endeavour focusing on the closed-form distributed parameter system of Euler-Bernoulli bimorph beam for investigating the FRFs under variable load resistance has been investigated by Erturk and Inman [16]. Wickenheiser and Garcia [17] further developed the analytical modelling of piezoelectric bimorph beam with magnetic tip mass located near to the fixed ferromagnetic structures in order to magnify the driving frequency below the fundamental frequency of the system. Moreover, development of the piezoelectric bimorph beam with tip mass under multidirectional excitation and under the strain field effects of the transverse and initial longitudinal forms have been explored by Lumentut and Howard 
[18]-[20] to investigate a broad range of case studies using mathematical studies of the orthonormalised electromechanical weak-form technique and closed-form boundary value method. In scrutinising the system response of the single piezoelectric beam given the previous literatures, the typical optimal power output mostly depends on the first mode. For this case, Lumentut et al [21] investigated the strategy of increasing power output and widening multifrequency band using the orthonormalised multielectromechanical dynamic equations reduced from the closed-form boundary value methods. Various parametric case studies have been developed using new electromechanical multimode FRFs of multiple piezoelectric bimorph beams connected in the series, parallel, and mixed series-parallel connections.

The following finite element literatures concern the use of piezoelectric components as smart material systems and have shown a variety of engineering applications, most notable of which has been devoted to ultrasonic transducers and active control systems either with feedback gain control or sensing and actuation response systems. For the current research, these piezoelectric finite element models, however, give the relevancy and basis that allow the development of the new numerical techniques for modelling the electromechanical power harvester as presented in this paper. In the ultrasonic transducer application, Allik and Hughes [22] and Allik et al [23] first introduced mathematical models of the linear constitutive equations of the three dimensional finite element piezoelectric vibration model using variational methods. Later on, comprehensive mechanical discretised elements for analysing an ultrasonic piezoelectric rod resonator [24] and the phenomenological electro-acoustic modelling for ultrasonic probes [25] were developed. Further application of the piezoelectric finite element has been developed for the active dynamic control system using the feedback gain control. Tzou and Tseng [26] developed the active dynamic control matrix equations of the distributed piezoelectric components onto plate structures using the Hamiltonian principle and Guyan reduction technique. Other published research works for analysing the controlled transient response [27]-[29] and transfer function [30] behaviours have been further investigated using the negative velocity feedback control system. For sensing and actuation response systems, the actuation system response using applied voltage into the piezoelectric actuator activated the piezoelectric sensor to create voltage output and deformation of the laminated composite structures [31]-[33]. More recently, the shunted electrical circuit connected to the piezoelectric patches has been formulated by Thomas et al [34] using finite element control system of the beam structure. Since the actual piezoelectric components are covered with electrode layers, most notably the piezoelectric finite element literatures ignore the electrode effect by taking the assumption that the electrical voltages for each element and nodes are independent. Only a few of the published papers related to the control finite element modelling have taken into consideration of the electrode effect by 
assuming equal voltage distribution on each piezoelectric element [29 31, 34]. However, the previous works are only based on the mechanical discretisation without considering the electrical discretised elements due to the effect of electrode layers. Ignoring the electrical discretised elements from the electrode layer can affect the electromechanical FRF of the piezoelectric power harvester using the closed circuit system. Also, there are no other previous research works for investigating the electromechanical finite element frequency responses using the electromechanical discretised elements under base excitation.

In the earlier studies of the electromechanical finite element response of piezoelectric power harvesting, Lumentut et al [35] initially developed an energy harvesting plate structure with the segmented piezoelectric element based on the Love-Kirchhoff plate theory for investigating the frequency and transient response behaviours. However, at the earlier stage, this model only considers the voltage output from the piezoelectric element without including the electrode effect with complete derivation of the electromechanical discretisation related to the system response. Later on, DeMarqui et al [36] further presented the cantilevered plate with the distributed piezoelectric layers by including the electrode effect as validated with the existing literature of the piezoelectric bimorph with tip mass presented by Erturk and Inman [16]. However, their proposed finite element model does not take into account the important physical issues of the electromechanical orthonormalised global matrix and scalar forms and the mathematical techniques of electromechanical discretisation. Aladwani et al [37] conducted further investigation of the dynamic magnifier using a spring system attached at the base structure of the cantilevered piezoelectric bimorph beam with tip mass. The system was used to model the optimised power harvesting frequency response behaviour. However, their proposed work neglects the electrode effect of the piezoelectric component for formulating the electromechanical discretised elements and also ignores the offset effect of the tip mass measured between the tip mass centroid and the end of the bimorph. Further research effort for investigating piezoelectric power harvesters has been developed using solid finite element-based ANSYS multiphysics software. Zhu et al [38] investigated prediction of power output FRFs under the resistive shunt circuit. Moreover, Yang and Tang [39] developed the electrical equivalent circuit model using SPICE software whose circuit parameters were adopted from ANSYS simulation for predicting the power output. However, although ANSYS software provides capabilities of three dimensional coupled field solid and plane elements, it does not provide the facility for modelling the laminated beam elements. For standard level of the energy harvesters, mesh generation using laminated beam elements is sufficient to model the laminated piezoelectric beam structures (e.g. unimorph, bimorph and multimorph).

In this paper, new numerical techniques of the electromechanical finite element vibration power harvester using the Euler-Bernoulli unimorph beam model are presented and validated with the 
experimental studies. The numerical models proposed here provide new technical contributions for analysing the interrelationship between coupled field equations, electromechanical discretisation, kinematic motions, Lagrangian electromechanical dynamic equations and electromechanical finite element equations. These technical contributions provide clear insight of presenting the new electromechanical finite element equations for power harvesting applications that can be highlighted in the following points.

- In considering the effect of electrode layers, the new electromechanical discretisation consisting of mechanical and electrical discretised elements is introduced to model the electromechanical finite element piezoelectric structure. The electromechanical discretised elements provide the important basis to formulate the FRFs using new electromechanical finite element dynamic equations.

- The elemental system dynamics of the piezoelectric unimorph beam including tip mass offset are reduced from the kinematic equations. It is found that the effect of the offset distance measured from the tip mass centroid and the end of the unimorph beam can result in extra terms into the mass matrix and the generalised input dynamic force vector. The benefit of applying the tip mass offset located after the end of the piezoelectric length is that it can provide a very practical MEMS power harvester design for any level of scalability. This can also avoid the use of material around the end of the piezoelectric length that can readily be damaged because of its brittle nature.

- The established new electromechanical finite element approach can be used conveniently for modelling different levels of scalability including the segmented piezoelectric element coupled with substructure and MEMS devices.

- The proposed numerical techniques also provide a complement to study the laminated piezoelectric beam with electrode layers in different applications.

- Lagrangian electromechanical dynamic equations are developed to formulate constitutive elemental matrices of the electromechanical finite element equations. The orthonormalised global matrices and global scalar forms can be further reduced to formulate new multimode electromechanical FRFs of the displacement, velocity, voltage, current, and power harvesting including optimal power harvester. The normalised strain node and eigenmode shapes are formulated using discretised elements for convergence studies. Finally, the parametric case studies with resistive shunt circuit based on the proposed novel electromechanical finite element and experimental validations are also presented and discussed.

\section{Coupled field equations of the piezoelectric energy harvester}

Constitutive linear piezoelectric beam equations can be formulated using the tensor electrical enthalpy concepts based on the continuum thermodynamics that can be condensed using Voigt's notation and 
Einstein's summation convention [19, 40-42]. The electrical enthalpy of the typical smart material with Lead Zirconate Titanate (PZT) can be formulated under adiabatic and isothermal processes as,

$$
\hat{H}\left(\boldsymbol{S}_{1}, \boldsymbol{E}_{3}\right)=\frac{1}{2} \bar{c}_{11}^{E} \boldsymbol{S}_{1} \boldsymbol{S}_{1}-e_{31} \boldsymbol{E}_{3} \boldsymbol{S}_{1}-\frac{1}{2} \varepsilon_{33}^{S} \boldsymbol{E}_{3} \boldsymbol{E}_{3}
$$

Notations of piezoelectric parameters used here are stated in accordance with IEEE standards [43]. Here the parameters $\bar{c}_{11}^{E}, e_{31}, \varepsilon_{33}^{S}, \boldsymbol{E}_{3}, \boldsymbol{T}_{1}$, and $\boldsymbol{S}_{1}$ represent the piezoelectric elastic stiffness at constant electric field, piezoelectric coefficient, permittivity under constant strain, electric field, stress, and strain, respectively. The parameter $\underset{\varepsilon_{33}}{S}=\varepsilon_{33}^{T}-e_{31} d_{31}$ or $\varepsilon_{33}^{S}=\varepsilon_{33}^{T}-d_{31}{ }^{2} \bar{c}_{11}^{E}$ is given where $\bar{c}_{11}^{E}=1 / s_{11}^{E}$ and $\varepsilon_{33}^{T}$ is the permittivity at constant stress and $s_{11}^{E}$ is the elastic compliance at constant electric field. Moreover, the common piezoelectric constant produced from the manufacturing company is in the form $d_{31}$ where this can be modified by multiplying the plane stress-based elastic stiffness at constant electric field to give $e_{31}=d_{31} \bar{c}_{11}^{E}$. The electric displacement of the piezoelectric element $\boldsymbol{D}_{3}$ can be obtained by differentiating equation (1) with respect to $\boldsymbol{E}_{3}$. This electric displacement of piezoelectricity can be formulated as,

$$
\boldsymbol{D}_{3}=-\frac{\partial \hat{H}}{\partial \boldsymbol{E}_{3}}=e_{31} \boldsymbol{S}_{1}+\varepsilon_{33}^{S} \boldsymbol{E}_{3}
$$

Note that for the convenient purpose in the forthcoming notations, the superscripts 1 and 2 being used in the parameters of stress, strain, elastic stiffness, density and cross-sectional area refer to the substructure and piezoelectric layers, respectively. Since the piezoelectric unimorph consists of the active and inactive layers, the plane stress field from the inactive or substructure layer can also be formulated as,

$$
\boldsymbol{T}_{1}^{(1)}=\bar{c}_{11}^{(1)} \boldsymbol{S}_{1}^{(1)}
$$

The piezoelectric stress field can also be formulated by differentiating equation (1) with respect to $\boldsymbol{S}_{1}$ to give,

$$
\boldsymbol{T}_{1}^{(2)}=\frac{\partial \hat{H}}{\partial \boldsymbol{S}_{1}^{(2)}}=\bar{c}_{11}^{(2)} \boldsymbol{S}_{1}^{(2)}-e_{31} \boldsymbol{E}_{3}
$$

Note that alternative derivations of equations (2) and (4) can also be obtained by using the enthalpy equation of state in terms of continuum thermopiezoelectricity, Maxwell's relations and Legendre's transformation [41, 44].

\section{Kinematic equations for elemental beam and arbitrary tip mass offset}

In this section, the kinematic motions for a continuous geometrical beam as shown in figure 1 are derived from the different configurations at a series of points, resulting in the deformations and 
velocity of the body. However, the kinematics for the tip mass offset whose motions are solely affected by the tip of the beam's transverse rectilinear $\dot{w}_{a b s}^{t i p}(L, t)$ and angular velocity $\dot{\theta}(L, t)$, is a rigid-body motion with different configurations that result in extra terms in the kinematic equations. In reviewing previous studies, the most recent published papers in the MEMS power harvesters [4550] included the tip mass offset without scrutinising the effect of the tip mass offset on the kinematic equations. The effect of the offset distance between the tip mass centroid and the end of the beam can effectively contribute to the mass matrix and the generalised input dynamic forces as shown in section 4.3. It is important to note here that the aim of formulating the kinematic equations is to formulate the Lagrangian functional energy forms that consist of the kinetic, potential and electrical energies of the piezoelectric unimorph with the tip mass as given in the forthcoming section.

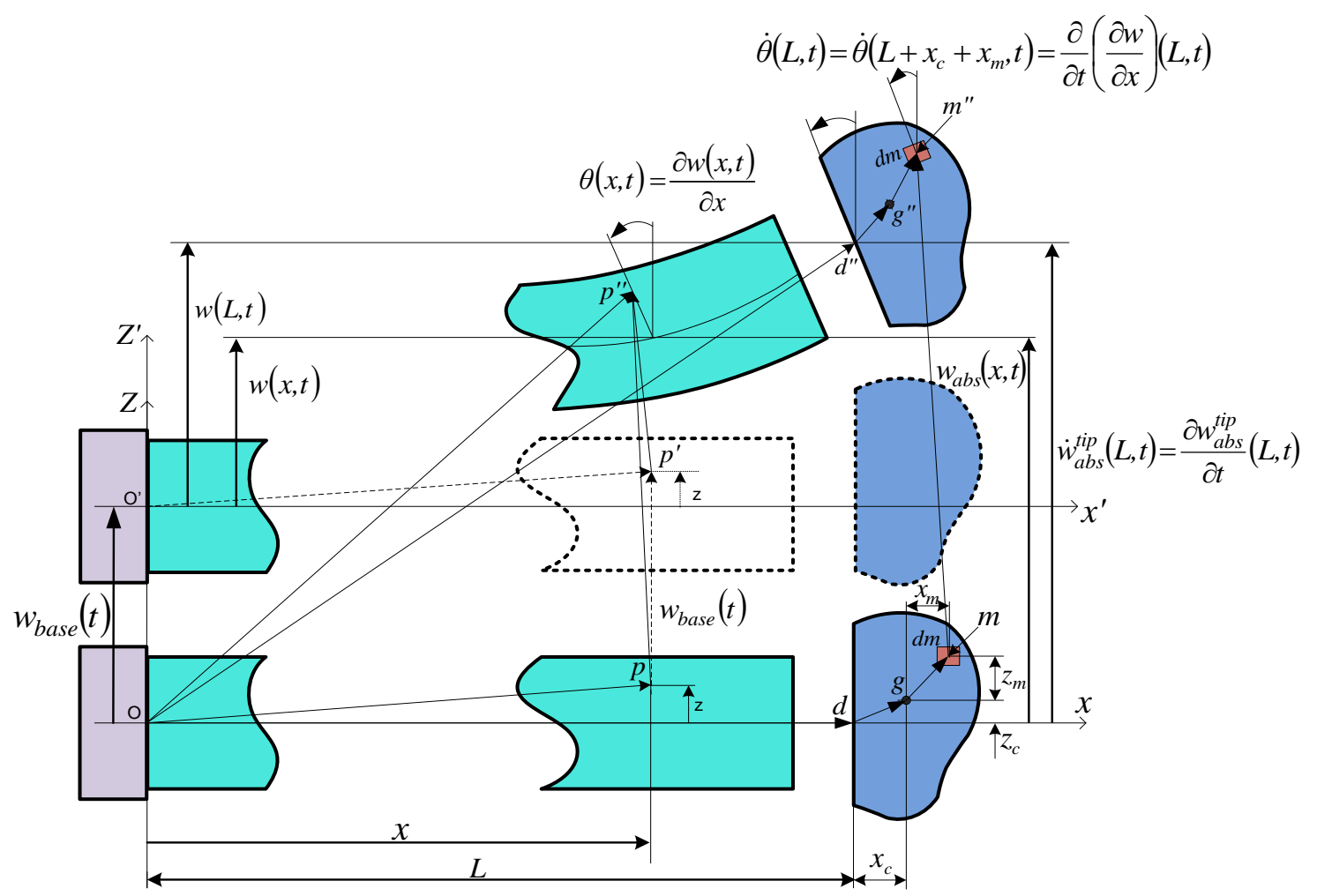

Figure 1. Kinematic motions of the beam and arbitrary tip mass offset.

In figure 1, the undeformed beam under base vector $w_{\text {base }}(t)$ moves from the fixed reference frame of $o X Z$ to the initial reference frame of $o^{\prime} X^{\prime} Z$. Since the base vector as the input excitation moves from point $o$ to $o^{\prime}$ at the designated reference frame, the position of point $p$ also moves to point $p^{\prime}$ with the same magnitude. As a result, the base vector creates the relative transverse deformation $w(x, t)$ 
measured from point $p^{\prime}$ moving to the final point $p^{\prime \prime}$. The absolute displacement $w_{a b s}(x, t)$ can also be obtained from the fixed reference frame of $o X Z$ to the final position. Note that the difference between the absolute displacement and the base vector defines the relative deformation. Moreover, the beam also carries an arbitrary tip mass offset whose kinematic motions must be considered. The position vectors of the tip mass start at point $d$ to the final differential element at point $m$ " where the resultant vectors at points $o-d^{\prime \prime}-g^{\prime \prime}-m^{\prime}$ can be defined. The point of attachment $d^{\prime \prime}$ (deformation point at the end of the beam) to the tip mass centroid at point $g^{\prime \prime}$ can be viewed as the offset of the tip mass. The origin of tip mass centroid can be determined from the differential element using the extra position vector from points $g^{\prime \prime}$ to $m^{\prime \prime}$. As mentioned previously, since the tip mass was assumed to be a rigid body, its relative motions depend on the tip of the beam's motion at point $d^{\prime \prime}$.

The position vector $\boldsymbol{R}^{p p^{\prime \prime}}$ for elemental beam can be defined as,

$$
\boldsymbol{R}^{p p^{\prime \prime}}(x, z, t)=\boldsymbol{R}^{o p^{\prime \prime}}-\boldsymbol{R}^{o p}
$$

where the position vectors $\boldsymbol{R}^{o p "}$ and $\boldsymbol{R}^{o p}$ can be written as,

$$
\boldsymbol{R}^{o p^{\prime \prime}}=(x-z \sin \theta(x, t)) \boldsymbol{e}_{1}+\left(w_{a b s}(x, t)+z \cos \theta(x, t)\right) \boldsymbol{e}_{3} \quad, \boldsymbol{R}^{o p}=x \boldsymbol{e}_{1}+z \boldsymbol{e}_{3}
$$

In simplification of the linear assumption, the small angle $\theta(x, t)=\partial w(x, t) / \partial x$ can be obtained after applying Taylor's theorem to give $\sin \theta(x, t) \approx \theta(x, t)$ and $\cos \theta(x, t) \approx 1$. As the initial position vector of the elemental beam $\boldsymbol{R}^{o p}$ is defined, the position vector $\boldsymbol{R}^{p p^{\prime \prime}}$ from equation (5) can be differentiated with respect to time to give,

$$
\dot{\boldsymbol{R}}^{p p^{\prime \prime}}(x, z, t)=\dot{\boldsymbol{R}}^{o p^{\prime \prime}}-\dot{\boldsymbol{R}}^{o p}=-\dot{\theta}(x, t) \boldsymbol{e}_{2} \times z \boldsymbol{e}_{3}+\left(\dot{w}_{\text {base }}(t)+\dot{w}(x, t)\right) \boldsymbol{e}_{3}
$$

It is noted that the absolute velocity vectors for the elemental beam and tip mass can be written as,

$$
\dot{\boldsymbol{w}}_{a b s}(x, t)=\left(\dot{w}_{\text {base }}(t)+\dot{w}(x, t)\right) \boldsymbol{e}_{3} \text { and } \dot{\boldsymbol{w}}_{a b s}^{t i p}(L, t)=\left(\dot{w}_{\text {base }}(t)+\dot{w}(L, t)\right) \boldsymbol{e}_{3}
$$

For the elemental tip mass, the position vector $\boldsymbol{R}^{\mathrm{mm}}$ can be formulated as,

$$
\boldsymbol{R}^{m m^{\prime \prime}}(x, z, t)=\boldsymbol{R}^{o d^{\prime \prime}}+\boldsymbol{R}^{d^{\prime \prime} g^{\prime \prime}}+\boldsymbol{R}^{g^{\prime \prime} m^{\prime \prime}}-\boldsymbol{R}^{o d}-\boldsymbol{R}^{d g}-\boldsymbol{R}^{g m}
$$

where each position vector can be formulated as,

$$
\begin{aligned}
\boldsymbol{R}^{\text {od }}=L \boldsymbol{e}_{1}, \boldsymbol{R}^{d g}=x_{c} \boldsymbol{e}_{1}+z_{c} \boldsymbol{e}_{3}, \boldsymbol{R}^{g m}=x_{m} \boldsymbol{e}_{1}+z_{m} \boldsymbol{e}_{3}, \boldsymbol{R}^{o d^{\prime \prime}}=L \boldsymbol{e}_{1}+\left(w_{b a s e}(t)+w(L, t)\right) \boldsymbol{e}_{3} \\
\boldsymbol{R}^{d^{\prime \prime} g^{\prime \prime}}=\left(x_{c} \cos \theta(L, t)-z_{c} \sin \theta(L, t)\right) \boldsymbol{e}_{1}+\left(z_{c} \cos \theta(L, t)+x_{c} \sin \theta(L, t)\right) \boldsymbol{e}_{3} \\
\boldsymbol{R}^{g^{\prime \prime} m^{\prime \prime}}=\left(x_{m} \cos \theta(L, t)-z_{m} \sin \theta(L, t)\right) \boldsymbol{e}_{1}+\left(z_{m} \cos \theta(L, t)+x_{m} \sin \theta(L, t)\right) \boldsymbol{e}_{3}
\end{aligned}
$$


Again, the linear system for the small angle $\theta(L, t)=\partial w(L, t) / \partial x$ can be made using Taylor's theorem to give $\sin \theta(L, t) \approx \theta(L, t)$ and $\cos \theta(L, t) \approx 1$. For this case, the velocity of the elemental tip mass can be obtained by differentiating equation (9) with respect to time to yield,

$$
\begin{aligned}
\dot{\boldsymbol{R}}^{m m^{\prime \prime}}(L, z, t) & =\dot{\boldsymbol{R}}^{o d^{\prime \prime}}+\dot{\boldsymbol{R}}^{d " g "}+\dot{\boldsymbol{R}}^{g " m^{\prime \prime}}-\dot{\boldsymbol{R}}^{o d}-\dot{\boldsymbol{R}}^{d g}-\dot{\boldsymbol{R}}^{g m} \\
& =\left(\dot{w}_{\text {base }}(t)+\dot{w}(L, t)\right) \boldsymbol{e}_{3}-\dot{\theta}(L, t) \boldsymbol{e}_{2} \times\left(z_{c} \boldsymbol{e}_{3}+x_{c} \boldsymbol{e}_{1}\right)-\dot{\theta}(L, t) \boldsymbol{e}_{2} \times\left(z_{m} \boldsymbol{e}_{3}+x_{m} \boldsymbol{e}_{1}\right) .
\end{aligned}
$$

The position vector $\boldsymbol{R}^{p^{\prime} p^{\prime}}$ can be specified as the relative displacement with respect to the moving base support from reference frame $o X Z$ to $o^{\prime} X^{\prime} Z^{\prime}$ as,

$$
\boldsymbol{R}^{p^{\prime} p^{\prime \prime}}(x, z, t)=\boldsymbol{R}^{p p^{\prime \prime}}-\boldsymbol{R}^{p p^{\prime}}=-z \theta(x, t) \boldsymbol{e}_{1}+w(x, t) \boldsymbol{e}_{3} .
$$

The elemental beam strain can be obtained by differentiating $\boldsymbol{R}^{p^{\prime} p^{\prime \prime}}$ with respect to $x$ giving the typical Euler-Bernoulli Beam theory as,

$$
S_{1}=\frac{\partial\left(\boldsymbol{R}^{\left.p^{\prime} p^{\prime \prime} \cdot \boldsymbol{e}_{1}\right)}\right.}{\partial x}=-z \frac{\partial^{2} w(x, t)}{\partial x^{2}} .
$$

Since the piezoelectric unimorph beam is viewed as the asymmetric structure with different material properties and geometrical dimensions, the asymmetric neutral axis [51] must be determined as given in appendix A.

\section{Electromechanical finite element vibration modelling of the piezoelectric energy harvester}

In the common practice, the surfaces of actual or physical piezoelectric material available in the market are covered evenly with thin conducting electrodes, resulting in the single voltage output during dynamic motions. As shown in figure 2(a), the physical structure of the unimorph beam with an arbitrary tip mass offset is chosen where it consists of piezoelectric material with conducting electrode layer and the substructure. However, in figure 2(b), the electromechanical discretisation consisting of mechanical and electrical discretised finite-elements is completely different from the physical structure. In this situation, the electromechanical finite element discretisation of the piezoelectric structure should be analysed using the generalised multi-output electrical current from each element connected with electrical parallel connection. Since the polarisation is proportional to the stress field and the stress field is also proportional to the strain field $[40,44,52]$, the polarisation $\boldsymbol{P}$ and electric field $\boldsymbol{E}$ directions are chosen in the 3-direction (thickness) along the z-axis. 

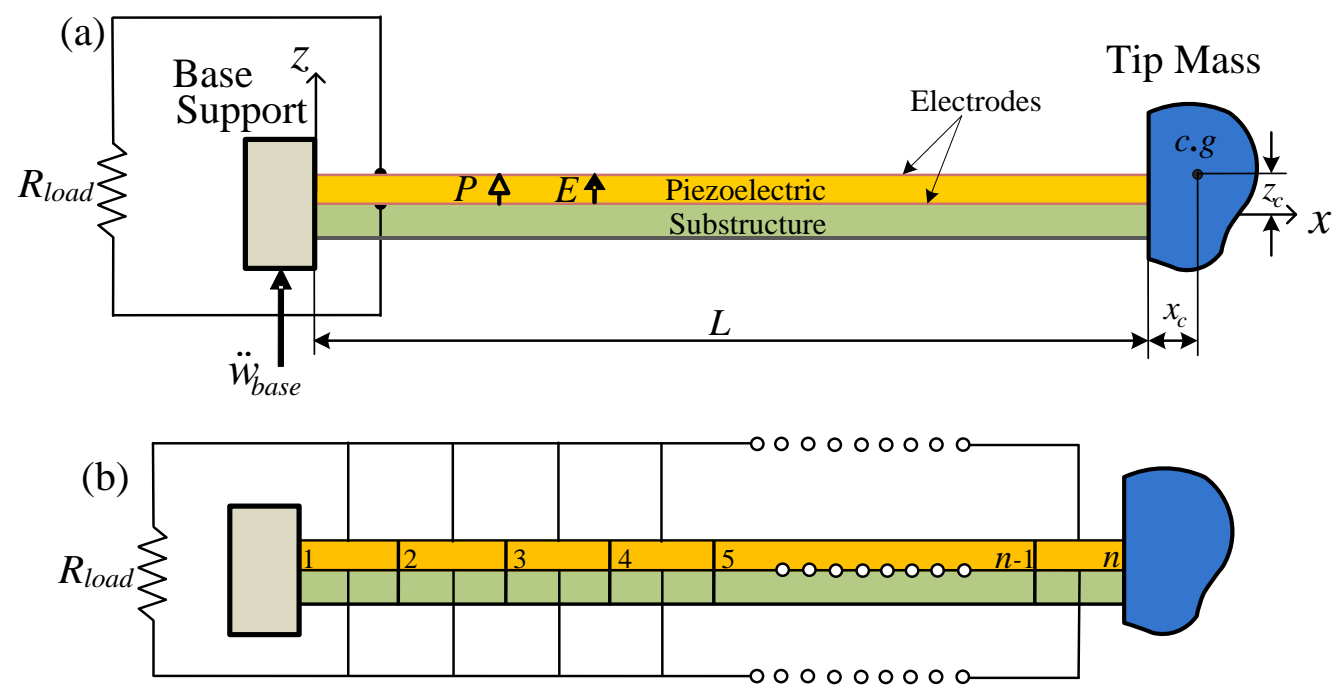

Figure 2. Electromechanical model of the unimorph beam with arbitrary tip mass offset under input base excitation based on (a) physical system connected electrically with the load resistance and (b) new electromechanical finite-element discretisation.

\subsection{Geometrical analysis of electromechanical element}

The first-order Hermite interpolation of the cubic displacement function is chosen here to formulate the unknown four degrees of freedom for the two node unimorph beam element [53-55]. The cubic displacement function must satisfy the beam theory continuity conditions for the translation and rotational displacements for the general location of the elements. It is important to note here that the kinematic equations given in section 3 can be used to formulate the local element near to the tip mass as shown in figure 3 , since the translation and angular velocities of the tip mass rigid body depend on the end of the unimorph beam of length, giving a particular insight to formulate the complete electromechanical finite element equations.

The first-order Hermite interpolation in terms of the polynomial $\Phi^{(1)}(x)$ and the unknown nodal displacement $u_{i}(t)$ for the elemental beam can be formulated as,

$$
w(x, t)=\sum_{i=1}^{2} \sum_{k=0}^{1} \Phi_{k i}^{(1)}(x) u_{i}^{(k)}(t)
$$

The property of Hermite polynomials for modelling the translation and rotation at the nodes can be formulated in terms of the $l$-derivatives to give,

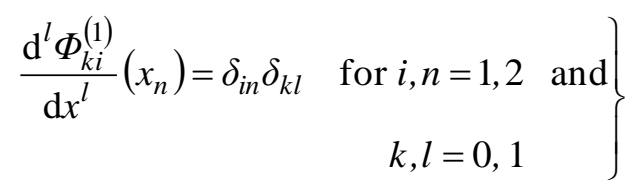




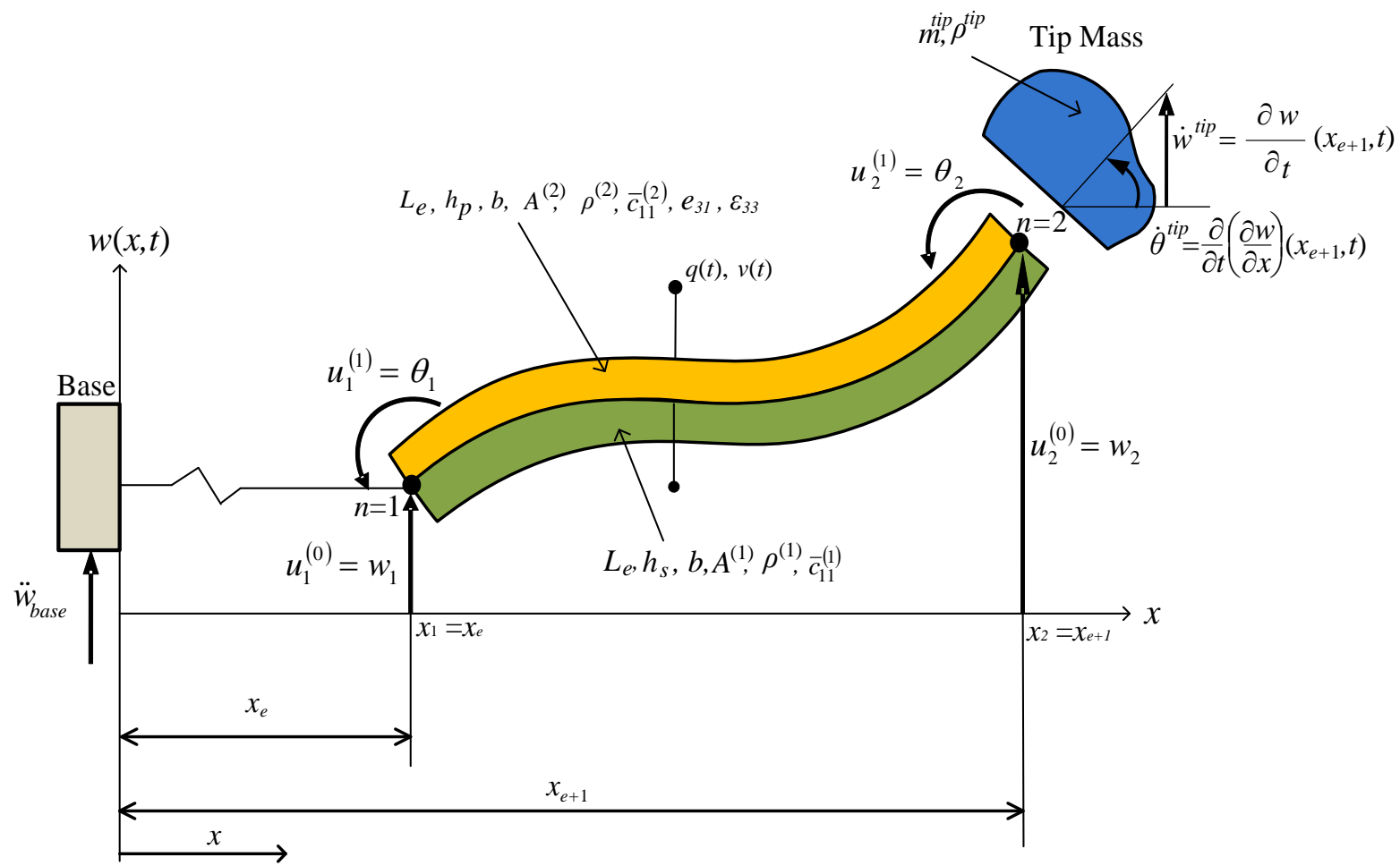

Figure 3. First-order Hermite polynomials for local unimorph element with an arbitrary tip mass at arbitrary nodes $n-1$ and $\mathrm{n}$ based on figure 2: by taking the example for $n=2$.

where $x_{n}$ is the location at $n$-th node and $\delta_{p q}$ is the Kronecker delta with the property as,

$$
\delta_{p q}=\left\{\begin{array}{l}
0 \text { if } p \neq q \\
1 \text { if } p=q
\end{array}\right.
$$

Corresponding to equations (15) and (16), the $l$-th derivatives of $w(x, t)$ can be written as,

$$
\frac{\mathrm{d}^{l} w_{n}}{\mathrm{~d} x^{l}}(x, t)=\sum_{i=1}^{2} \sum_{k=0}^{1} \frac{\mathrm{d}^{l} \Phi_{k i}^{(1)}}{\mathrm{d} x^{l}}\left(x_{n}\right) u_{i}^{(k)}(t)
$$

or it can be formulated in terms of the unknown nodal displacement forms as,

$$
\frac{\mathrm{d}^{l} w_{n}}{\mathrm{~d} x^{l}}(t)=\sum_{i=1}^{2} \sum_{k=0}^{1} \delta_{i n} \delta_{k l} u_{i}^{(k)}(t)=u_{n}^{(l)}(t)
$$

Note that equation (18) clearly shows certain physical meaning after applying equations (15) and (16), giving the nodal displacement forms as a function of time. Corresponding to index notation from equation (15), equation (14) can be modified by applying equation (18) to give,

$$
w(x, t)=\sum_{i=1}^{2} \sum_{k=0}^{1} \Phi_{k i}^{(1)}(x) \frac{\mathrm{d}^{k} w_{i}}{\mathrm{~d} x^{k}}(t)
$$


To find four terms of first-order Hermite polynomials, the property given from equation (15) can be used to identify four elemental boundary conditions for each degree of freedom at the nodes. In such condition, the cubic equations with four unknown coefficients for each degree of freedom at the nodes can be used and the results of the Hermite polynomials can be formulated after simplifying to give,

$$
\begin{aligned}
\Phi_{01}^{(1)}(x)=1-3\left(\frac{x-x_{e}}{L_{e}}\right)^{2}+2\left(\frac{x-x_{e}}{L_{e}}\right)^{3}, & \Phi_{11}^{(1)}(x)=\left(x-x_{e}\right)\left(1-\frac{x-x_{e}}{L_{e}}\right)^{2} \\
\Phi_{02}^{(1)}(x)=3\left(\frac{x-x_{e}}{L_{e}}\right)^{2}-2\left(\frac{x-x_{e}}{L_{e}}\right)^{3}, & \Phi_{12}^{(1)}(x)=\left(x-x_{e}\right)\left[\left(\frac{x-x_{e}}{L_{e}}\right)^{2}-\frac{x-x_{e}}{L_{e}}\right] \\
L_{e} & =x_{e+1}-x_{e}
\end{aligned}
$$

Corresponding to equation (14), the simplified first-order Hermite interpolation function of the unimorph beam can be modified into a matrix form to give,

$$
\boldsymbol{w}(x, t)=\boldsymbol{\Phi}^{e}(x) \boldsymbol{u}^{e}(t)
$$

where

$$
\begin{aligned}
& \boldsymbol{\Phi}^{e}(x)= {\left[\begin{array}{llll}
\Phi_{1}(x) & \Phi_{2}(x) & \Phi_{3}(x) & \Phi_{4}(x)
\end{array}\right] } \\
& \boldsymbol{u}^{e}(t)=\left[\begin{array}{llll}
u_{1} & u_{2} & u_{3} & u_{4}
\end{array}\right]^{T}
\end{aligned}
$$

Each parameter from equation (22) can be written in terms of the elemental displacement vector $\boldsymbol{u}$ and shape function $\Phi$ for each node as,

$$
\begin{gathered}
u_{1}(t)=u_{1}^{(0)}(t)=w_{1}(t), \quad u_{2}(t)=u_{1}^{(1)}(t)=\theta_{1}(t), \quad u_{3}(t)=u_{2}^{(0)}(t)=w_{2}(t), \quad u_{4}(t)=u_{2}^{(1)}(t)=\theta_{2}(t) \\
\Phi_{1}(x)=\Phi_{01}^{(1)}(x), \quad \Phi_{2}(x)=\Phi_{11}^{(1)}(x), \quad \Phi_{3}(x)=\Phi_{02}^{(1)}(x), \quad \Phi_{4}(x)=\Phi_{12}^{(1)}(x)
\end{gathered}
$$

Corresponding to equation (13), the strain-displacement relationship in terms of the differential form of the shape function of the piezoelectric unimorph beam can be formulated by substituting equation (21) to give,

$$
\boldsymbol{S}(x, t)=-z \boldsymbol{\Psi}^{e}(x) \boldsymbol{u}^{e}(t)
$$

where the differential form of the shape function of the strain displacement relationship can be formulated as,

where

$$
\begin{aligned}
& \boldsymbol{\Psi}^{e}(x)=\frac{\mathrm{d}^{2} \boldsymbol{\Phi}^{e}(x)}{\mathrm{d} x^{2}}=\left[\begin{array}{llll}
\Psi_{1}(x) & \Psi_{2}(x) & \Psi_{3}(x) & \Psi_{4}(x)
\end{array}\right] \\
& \Psi_{1}(x)=-\frac{6}{L_{e}{ }^{2}}+\frac{12\left(x-x_{e}\right)}{L_{e}{ }^{3}} \quad, \quad \Psi_{2}(x)=\frac{6\left(x-x_{e}\right)}{L_{e}{ }^{2}}-\frac{4}{L_{e}}
\end{aligned}
$$




$$
\Psi_{3}(x)=-\frac{12\left(x-x_{e}\right)}{L_{e}{ }^{3}}+\frac{6}{L_{e}{ }^{2}} \quad, \quad \Psi_{4}(x)=\frac{6\left(x-x_{e}\right)}{L_{e}{ }^{2}}-\frac{2}{L_{e}}
$$

The elemental discretised electric field $\boldsymbol{E}$, induced by the strain field due to input ambient vibration, can create a polarisation in the piezoelectric material in the $z$-direction along its thickness generating the electrical voltages. The electrical field is a function of the electrical potential with negative gradient operator given by,

$$
\boldsymbol{E}_{3}=-\nabla \boldsymbol{\phi}^{e}(z, t)
$$

where $\boldsymbol{\phi}^{e}(z, t)=\boldsymbol{\vartheta}^{e}(z) \boldsymbol{v}^{e}(t)$ is the electrical potential with linear assumption and the parameter $\vartheta^{e}(z)=\left(z-z_{n}+h_{p}\right) / h_{p}$ is the shape function over the interval $z_{n}-h_{p} \leq z \leq z_{n}$ and $z_{n}$ indicates the distance from the asymmetric neutral axis as given in appendix A. Symbol $\nabla$ is a gradient operator for the first derivative of the shape function with respect to the thickness direction, giving $\boldsymbol{\Omega}^{(e)}(z)=d \boldsymbol{\vartheta}^{e}(z) / d z=1 / h_{p}$. On this case, the discretised electric field can be reformulated as,

$$
\boldsymbol{E}_{3}=-\boldsymbol{\Omega}^{e}(z) \boldsymbol{v}^{(e)}(t)
$$

The expression of the stress field in the partial differential shape function form can be formulated for both piezoelectric and substructure elements by substituting equations (24) and (28) into equations (3) and (4) to give,

$$
\begin{gathered}
\boldsymbol{T}_{1}^{(2)}=-z \bar{c}_{11}^{(2)} \boldsymbol{\Psi}^{e}(x) \boldsymbol{u}^{e}(t)+e_{31} \boldsymbol{\Omega}^{e}(z) \boldsymbol{v}^{e}(t) \\
\boldsymbol{T}_{1}^{(1)}=-z \bar{c}_{11}^{(1)} \boldsymbol{\Psi}^{e}(x) \boldsymbol{u}^{e}(t)
\end{gathered}
$$

The electric displacement vector of the piezoelectric component can be formulated by substituting equations (24) and (28) into (2) to give,

$$
\boldsymbol{D}_{3}=z e_{31} \boldsymbol{\Psi}^{e}(x) \boldsymbol{u}^{e}(t)-\varepsilon_{33}^{S} \boldsymbol{\Omega}^{e}(z) \boldsymbol{v}^{e}(t)
$$

\subsection{Lagrangian electromechanical finite element equation}

Constitutive electromechanical finite element dynamic equations are developed for modelling the elemental piezoelectric unimorph with the tip mass offset under input base transverse excitation. The functional energy forms using the extended Lagrangian formulation consist of kinetic $K E$, potential $P E$ and electrical $W E$ energies and non-conservative work $W F$. The extended Lagrange equation can be written as,

$$
\sum_{s=1}\left[\frac{\mathrm{d}}{\mathrm{d} t} \frac{\partial \Pi}{\partial \boldsymbol{\eta}_{s}}-\frac{\partial \Pi}{\partial \boldsymbol{\eta}_{s}}-\boldsymbol{F}_{s}\right]=0
$$

where $\forall \Pi=K E-P E+W E, \eta_{s} \in\{\dot{u}(t), u(t), v(t)\}$ 
and $\quad \boldsymbol{F}_{s} \in\left\{\ddot{w}_{\text {base }}(t), q(t)\right\}$ or $\left\{\sum_{s=1} F_{s}\right\}=\left\{\frac{\partial W F}{\partial u^{e}(t)}+\frac{\partial W F}{\partial v^{e}(t)}\right\}$

The kinetic energy can be formulated based on the mass density of the piezoelectric unimorph and density of tip mass as,

$$
\begin{aligned}
K E= & \frac{1}{2} \int_{x_{e}}^{x_{e+1}} \int_{A^{(1)}} z^{2} \rho^{(1)} \dot{\boldsymbol{\theta}}(x, t)^{T} \dot{\boldsymbol{\theta}}(x, t) \mathrm{d} A^{(1)} \mathrm{d} x+\frac{1}{2} \int_{x_{e}}^{x_{e+1}} \int_{A^{(2)}} z^{2} \rho^{(2)} \dot{\boldsymbol{\theta}}(x, t)^{T} \dot{\boldsymbol{\theta}}(x, t) \mathrm{d} A^{(2)} \mathrm{d} x \\
& +\frac{1}{2} \int_{x_{e}}^{x_{e+1}} \int_{A^{(1)}} \rho^{(1)} \dot{\boldsymbol{w}}(x, t)^{T} \dot{\boldsymbol{w}}(x, t) \mathrm{d} A^{(1)} \mathrm{d} x+\frac{1}{2} \int_{x_{e}}^{x_{e+1}} \int_{A^{(2)}} \rho^{(2)} \dot{\boldsymbol{w}}(x, t)^{T} \dot{\boldsymbol{w}}(x, t) \mathrm{d} A^{(2)} \mathrm{d} x \\
& +I_{0}^{t i p} x_{c} \dot{\boldsymbol{w}}\left(x_{e+1}, t\right)^{T} \dot{\boldsymbol{\theta}}\left(x_{e+1}, t\right)+\frac{1}{2} I_{0}^{t i p} \dot{\boldsymbol{w}}\left(x_{e+1}, t\right)^{T} \dot{\boldsymbol{w}}\left(x_{e+1}, t\right)+\frac{1}{2} I_{2}^{t i p} \dot{\boldsymbol{\theta}}\left(x_{e+1}, t\right)^{T} \dot{\boldsymbol{\theta}}\left(x_{e+1}, t\right)
\end{aligned}
$$

Derivation of equation (32) can be seen in appendix B. The potential energy for the piezoelectric unimorph can be formulated as,

$$
P E=\frac{1}{2} \int_{x_{e}}^{x_{e+1}} \int_{A^{(1)}} S_{1}^{(1)^{T}} \boldsymbol{T}_{1}^{(1)} \mathrm{d} A^{(1)} \mathrm{d} x+\frac{1}{2} \int_{x_{e}}^{x_{e+1}} \int_{A^{(2)}} S_{1}^{(2)^{T}} \boldsymbol{T}_{1}^{(2)} \mathrm{d} A^{(2)} \mathrm{d} x
$$

The electrical energy term for the piezoelectric layer can be reduced as,

$$
W E=\frac{1}{2} \int_{x_{e}}^{x_{e+1}} \int_{A^{(2)}} \boldsymbol{E}_{3}^{T} \boldsymbol{D}_{3} \mathrm{~d} A^{(2)} \mathrm{d} x
$$

The non-conservative work on the system due to the input base excitation and electrical charge output can be written as,

$$
\begin{aligned}
W F=- & \int_{x_{e}}^{x_{e+1}} \int_{A^{(1)}} \rho^{(1)} \boldsymbol{w}(x, t)^{T} \mathrm{~d} A^{(1)} \mathrm{d} x \ddot{\boldsymbol{w}}_{\text {base }}(t)-\int_{x_{e}}^{x_{e+1}} \int_{A^{(2)}} \rho^{(2)} \boldsymbol{w}(x, t)^{T} \mathrm{~d} A^{(2)} \mathrm{d} x \ddot{\boldsymbol{w}}_{\text {base }}(t) \\
& \quad-I_{0}^{t i p} x_{c} \boldsymbol{\theta}\left(x_{e+1}, t\right)^{T} \ddot{\boldsymbol{w}}_{\text {base }}(t)-I_{0}^{t i p} \boldsymbol{w}\left(x_{e+1}, t\right)^{T} \ddot{\boldsymbol{w}}_{\text {base }}(t)+\boldsymbol{q}^{(e)}(t) \boldsymbol{v}^{(e)}(t)
\end{aligned}
$$

The reduced form of equation (35) due to the input base excitation can be found in appendix $\mathrm{C}$. The expressions of the functional energy forms including external work can be modified in terms of the discretised mechanical and electrical shape functions. The extended kinetic energy form can be formulated by substituting equations (21) into equation (32) giving,

$$
\begin{aligned}
K E= & \frac{1}{2} \int_{x_{e}}^{x_{e+1}} \int_{A^{(1)}} z^{2} \rho^{(1)} \dot{\boldsymbol{u}}^{e}(t)^{T} \frac{\mathrm{d} \boldsymbol{\Phi}^{e}(x)^{T}}{\mathrm{~d} x} \frac{\mathrm{d} \boldsymbol{\Phi}^{e}(x)}{\mathrm{d} x} \dot{\boldsymbol{u}}^{e}(t) \mathrm{d} A^{(1)} \mathrm{d} x+\frac{1}{2} \int_{x_{e}}^{x_{e+1}} \int_{A^{(2)}} z^{2} \rho^{(2)} \dot{\boldsymbol{u}}^{e}(t)^{T} \frac{\mathrm{d} \boldsymbol{\Phi}^{e}(x)^{T}}{\mathrm{~d} x} \frac{\mathrm{d} \boldsymbol{\Phi}^{e}(x)}{\mathrm{d} x} \dot{\boldsymbol{u}}^{e}(t) \mathrm{d} A^{(2)} \mathrm{d} x \\
& +\frac{1}{2} \int_{x_{e}}^{x_{e+1}} \int_{A^{(1)}} \rho^{(1)} \dot{\boldsymbol{u}}^{e}(t)^{T} \boldsymbol{\Phi}^{e}(x)^{T} \boldsymbol{\Phi}^{(e)}(x) \dot{\boldsymbol{u}}^{(e)}(t) \mathrm{d} A^{(1)} \mathrm{d} x+\frac{1}{2} \int_{x_{e}}^{x_{e+1}} \int_{A^{(2)}} \rho^{(2)} \dot{\boldsymbol{u}}^{e}(t)^{T} \boldsymbol{\Phi}^{e}(x)^{T} \boldsymbol{\Phi}^{e}(x) \dot{\boldsymbol{u}}^{e}(t) \mathrm{d} A^{(2)} \mathrm{d} x \\
& +I_{0}^{t i p} x_{c} \dot{\boldsymbol{u}}(t)^{T} \boldsymbol{\Phi}^{e}\left(x_{e+1}\right)^{T} \frac{d \boldsymbol{\Phi}^{e}\left(x_{e+1}\right)}{d x} \dot{\boldsymbol{u}}(t)+\frac{1}{2} I_{0}^{t i p} \dot{\boldsymbol{u}}^{e}(t)^{T} \boldsymbol{\Phi}^{e}\left(x_{e+1}\right)^{T} \boldsymbol{\Phi}^{e}\left(x_{e+1}\right) \dot{\boldsymbol{u}}^{e}(t)
\end{aligned}
$$




$$
+\frac{1}{2} I_{2}^{t i p} \frac{d \boldsymbol{\Phi}^{e}\left(x_{e+1}\right)^{T}}{d x} \dot{\boldsymbol{u}}^{e}(t)^{T} \frac{d \Phi^{e}\left(x_{e+1}\right)}{d x} \dot{\boldsymbol{u}}^{e}(t)
$$

Corresponding to equations (24) and (29), the extended potential energy form can be reduced as,

$$
\begin{aligned}
P E= & \frac{1}{2} \int_{x_{e}}^{x_{e+1}} \int_{A^{(1)}} z^{2} \boldsymbol{u}^{e}(t)^{T} \boldsymbol{\Psi}^{e}(x)^{T} \bar{c}_{11}^{(1)} \boldsymbol{\Psi}^{e}(x) \boldsymbol{u}^{e}(t) \mathrm{d} A^{(1)} \mathrm{d} x+\frac{1}{2} \int_{x_{e}}^{x_{e+1}} \int_{A^{(2)}} z^{2} \boldsymbol{u}^{e}(t)^{T} \boldsymbol{\Psi}^{e}(x)^{T} \bar{c}_{11}^{(2)} \boldsymbol{\Psi}^{e}(x) \boldsymbol{u}^{e}(t) \mathrm{d} A^{(2)} \mathrm{d} x \\
& -\frac{1}{2} \int_{x_{e}}^{x_{e+1}} \int_{A^{(2)}} z e_{31} \boldsymbol{u}^{e}(t)^{T} \boldsymbol{\Psi}^{e}(x)^{T} \boldsymbol{\Omega}^{e}(z) \boldsymbol{v}^{e}(t) \mathrm{d} A^{(2)} \mathrm{d} x
\end{aligned}
$$

The extended electrical energy expression can be reduced by substituting equations (28) and (30) into equation (34) as,

$$
W E=\frac{1}{2} \int_{x_{e}}^{x_{e+1}} \int_{A^{(2)}} z e_{31} \boldsymbol{v}^{e}(t)^{T} \boldsymbol{\Omega}^{e}(z)^{T} \boldsymbol{\Psi}^{e}(x) \boldsymbol{u}^{e}(t) \mathrm{d} A^{(2)} \mathrm{d} x+\frac{1}{2} \int_{x_{e}}^{x_{e+1}} \int_{A^{(2)}} \varepsilon_{33}^{S} \boldsymbol{v}^{e}(t)^{T} \boldsymbol{\Omega}^{e}(z)^{T} \boldsymbol{\Omega}^{e}(z) \boldsymbol{v}^{e}(t) \mathrm{d} A^{(2)} \mathrm{d} x
$$

The extended virtual work in terms of the expression of non-conservative energy can be formulated by substituting equations (21) into (35) to give,

$$
\begin{aligned}
W F= & -\int_{x_{e}}^{x_{e+1}} \int_{A^{(1)}} \rho^{(1)} \boldsymbol{\Phi}^{e}(x)^{T} \boldsymbol{u}^{e}(t) \mathrm{d} A^{(1)} \mathrm{d} x \ddot{\boldsymbol{w}}_{\text {base }}(t)-\int_{x_{e}}^{x_{e+1}} \int_{A^{(2)}} \rho^{(2)} \boldsymbol{\Phi}^{e}(x)^{T} \boldsymbol{u}^{e}(t) \mathrm{d} A^{(2)} \mathrm{d} x \ddot{\boldsymbol{w}}_{\text {base }}(t) \\
& -I_{0}^{t i p} x_{c} \frac{d \boldsymbol{\Phi}^{e}\left(x_{e+1}\right)^{T}}{d x} \boldsymbol{u}^{e}(t) \ddot{\boldsymbol{w}}_{\text {base }}(t)-I_{0}^{t i p} \boldsymbol{\Phi}^{e}\left(x_{e+1}\right) \boldsymbol{u}^{e}(t) \ddot{\boldsymbol{w}}_{\text {base }}(t)+\boldsymbol{q}^{e}(t) \boldsymbol{v}^{e}(t)
\end{aligned}
$$

The expressions given from equations (36)-(39) can be substituted into equation (31) to give two electromechanical dynamic equations. After simplifying, the first electromechanical dynamic equation due to the transverse bending form can be expressed as,

$$
\begin{aligned}
& \int_{x_{e}}^{x_{e+1}} \int_{A^{(1)}} z^{2} \frac{\mathrm{d} \boldsymbol{\Phi}^{e}(x)^{T}}{\mathrm{~d} x} \frac{\mathrm{d} \boldsymbol{\Phi}^{e}(x)}{\mathrm{d} x} \ddot{\boldsymbol{u}}^{e}(t) \mathrm{d} A^{(1)} \mathrm{d} x+\int_{x_{e}}^{x_{e+1}} \int_{A^{(2)}} z^{2} \frac{\mathrm{d} \boldsymbol{\Phi}^{e}(x)^{T}}{\mathrm{~d} x} \frac{\mathrm{d} \boldsymbol{\Phi}^{e}(x)}{\mathrm{d} x} \ddot{\boldsymbol{u}}^{e}(t) \mathrm{d} A^{(2)} \mathrm{d} x \\
& +\int_{x_{e}}^{x_{e+1}} \int_{A^{(1)}} \rho^{(1)} \boldsymbol{\Phi}^{e}(x)^{T} \boldsymbol{\Phi}^{e}(x) \ddot{\boldsymbol{u}}^{e}(t) \mathrm{d} A^{(1)} \mathrm{d} x+\int_{x_{e}}^{x_{e+1}} \int_{A^{(2)}} \rho^{(2)} \boldsymbol{\Phi}^{e}(x)^{T} \boldsymbol{\Phi}^{(e)}(x) \ddot{\boldsymbol{u}}^{(e)}(t) \mathrm{d} A^{(2)} \mathrm{d} x \\
& +2 I_{0}^{t i p} x_{c} \boldsymbol{\Phi}^{e}\left(x_{e+1}\right)^{T} \frac{\mathrm{d} \boldsymbol{\Phi}^{e}\left(x_{e+1}\right)}{\mathrm{d} x} \ddot{\boldsymbol{u}}(t)+I_{0}^{t i p} \boldsymbol{\Phi}^{e}\left(x_{e+1}\right)^{T} \boldsymbol{\Phi}^{e}\left(x_{e+1}\right) \ddot{\boldsymbol{u}}^{e}(t)+I_{2}^{t i p} \frac{\mathrm{d} \boldsymbol{\Phi}^{e}\left(x_{e+1}\right)^{T}}{\mathrm{~d} x} \frac{\mathrm{d} \boldsymbol{\Phi}^{e}\left(x_{e+1}\right)}{\mathrm{d} x} \ddot{\boldsymbol{u}}^{e}(t) \\
& +\int_{x_{e}}^{x_{e+1}} \int_{A^{(1)}} z^{2} \bar{c}_{11}^{(1)} \boldsymbol{\Psi}^{e}(x) \boldsymbol{\Psi}^{e}(x)^{T} \boldsymbol{u}^{e}(t) \mathrm{d} A^{(1)} \mathrm{d} x+\int_{x_{e}}^{x_{e+1}} \int_{A^{(2)}} z^{2} \bar{c}_{11}^{(2)} \boldsymbol{\Psi}^{e}(x) \boldsymbol{\Psi}^{e}(x)^{T} \boldsymbol{u}^{e}(t) \mathrm{d} A^{(2)} \mathrm{d} x \\
& -\frac{1}{2} \int_{x_{e}}^{x_{e+1}} \int_{A^{(2)}} z e_{31} \boldsymbol{\Psi}^{e}(x)^{T} \boldsymbol{\Omega}^{e}(z) \boldsymbol{v}^{e}(t) \mathrm{d} A^{(2)} \mathrm{d} x-\frac{1}{2} \int_{x_{e}}^{x_{e+1}} \int_{A^{(2)}} z e_{31} \boldsymbol{\Psi}^{e}(x)^{T} \boldsymbol{\Omega}^{e}(z) \boldsymbol{v}^{e}(t) \mathrm{d} A^{(2)} \mathrm{d} x
\end{aligned}
$$




$$
\begin{aligned}
& =-\int_{x_{e}}^{x_{e+1}} \int_{A^{(1)}} \rho^{(1)} \boldsymbol{\Phi}^{e}(x)^{T} \mathrm{~d} A^{(1)} \mathrm{d} x \ddot{\boldsymbol{w}}_{\text {base }}(t)-\int_{x_{e}}^{x_{e+1}} \int_{A^{(2)}} \rho^{(2)} \boldsymbol{\Phi}^{e}(x)^{T} \mathrm{~d} A^{(2)} \mathrm{d} x \ddot{\boldsymbol{w}}_{\text {base }}(t) \\
& -I_{0}^{t i p} x_{c} \frac{\mathrm{d} \boldsymbol{\Phi}^{e}\left(L_{e}\right)^{T}}{\mathrm{~d} x} \ddot{\boldsymbol{w}}_{\text {base }}(t)-I_{0}^{t i p} \boldsymbol{\Phi}^{e}\left(L_{e}\right)^{T} \ddot{\boldsymbol{w}}_{\text {base }}(t)
\end{aligned}
$$

The second electromechanical dynamic equation due to the electrical form can be expressed as,

$$
\begin{aligned}
& -\frac{1}{2} \int_{x_{e}}^{x_{e+1}} \int_{A^{(2)}} z e_{31} \boldsymbol{\Omega}^{e}(z)^{T} \boldsymbol{\Psi}^{e}(x) \boldsymbol{u}^{e}(t) \mathrm{d} A^{(2)} \mathrm{d} x-\frac{1}{2} \int_{x_{e}}^{x_{e+1}} \int_{A^{(2)}} z e_{31} \boldsymbol{\Omega}^{e}(z)^{T} \boldsymbol{\Psi}^{e}(x) \boldsymbol{u}^{e}(t) \mathrm{d} A^{(2)} \mathrm{d} x \\
& -\int_{x_{e}}^{x_{e+1}} \int_{A^{(2)}} \varepsilon_{33}^{S} \boldsymbol{\Omega}(z)^{T} \boldsymbol{\Omega}(z) \boldsymbol{v}(t) \mathrm{d} A^{(2)} \mathrm{d} x-\boldsymbol{q}^{e}(t)=0
\end{aligned}
$$

Equation (41) can be modified by differentiating with respect to time to give,

$$
\begin{aligned}
& -\frac{1}{2} \int_{x_{e}}^{x_{e+1}} \int_{A^{(2)}} z \boldsymbol{\Omega}(z)^{T} e_{31} \boldsymbol{\Psi}(x) \dot{\boldsymbol{u}}(t) \mathrm{d} A^{(2)} \mathrm{d} x-\frac{1}{2} \int_{x_{e}}^{x_{e+1}} \int_{A^{(2)}} z \boldsymbol{\Omega}(z)^{T} e_{31} \boldsymbol{\Psi}(x) \dot{\boldsymbol{u}}(t) \mathrm{d} A^{(2)} \mathrm{d} x \\
& -\int_{x_{e}}^{x_{e+1}} \int_{A^{(2)}} \varepsilon_{33}^{S} \boldsymbol{\Omega}(z)^{T} \boldsymbol{\Omega}(z) \dot{\boldsymbol{v}}(t) \mathrm{d} A^{(2)} \mathrm{d} x-\boldsymbol{i}_{p}^{e}(t)=0
\end{aligned}
$$

\subsection{Element matrices of electromechanical dynamic equations}

Constitutive electromechanical non-homogenous differential dynamic equations in terms of equations (40) and (42) can be arranged into matrix form by including Rayleigh damping to give,

$$
\left[\begin{array}{cc}
\boldsymbol{M}^{e} & 0 \\
0 & 0
\end{array}\right]\left\{\begin{array}{l}
\ddot{\boldsymbol{u}}^{e}(t) \\
\ddot{\boldsymbol{v}}^{e}(t)
\end{array}\right\}+\left[\begin{array}{cc}
\boldsymbol{C}^{e} & 0 \\
\boldsymbol{P}_{\theta}^{e^{T}} & \boldsymbol{P}_{D}^{e}
\end{array}\right]\left\{\begin{array}{l}
\dot{\boldsymbol{u}}^{e}(t) \\
\dot{\boldsymbol{v}}^{e}(t)
\end{array}\right\}+\left[\begin{array}{cc}
\boldsymbol{K}^{e} & \boldsymbol{P}_{\theta}^{e} \\
0 & 0
\end{array}\right]\left\{\begin{array}{l}
\boldsymbol{u}^{e}(t) \\
\boldsymbol{v}^{e}(t)
\end{array}\right\}=\left\{\begin{array}{l}
\boldsymbol{F}^{e} \\
\boldsymbol{i}_{p}^{e}
\end{array}\right\}
$$

where

$$
\begin{aligned}
& \boldsymbol{M}^{e}=\sum_{n l=1}\left(\int_{x_{e}}^{x_{e+1}} \int_{(n l)} z^{2} \rho^{(n l)} \frac{\mathrm{d} \boldsymbol{\Phi}^{e}(x)^{T}}{\mathrm{~d} x} \frac{\mathrm{d} \boldsymbol{\Phi}^{e}(x)}{\mathrm{d} x} \mathrm{~d} A^{(n l)} \mathrm{d} x\right)+\sum_{n l=1}\left(\int_{x_{e}}^{x_{e+1}} \int_{A^{(n l)}} \rho^{(n l)} \boldsymbol{\Phi}^{e}(x)^{T} \boldsymbol{\Phi}^{e}(x) \mathrm{d} A^{(n l)} \mathrm{d} x\right) \\
& +2 I_{0}^{t i p} x_{c} \boldsymbol{\Phi}^{e}\left(x_{e+1}\right)^{T} \frac{\mathrm{d} \boldsymbol{\Phi}^{e}\left(x_{e+1}\right)}{\mathrm{d} x}+I_{0}^{t i p} \boldsymbol{\Phi}^{e}\left(x_{e+1}\right)^{T} \boldsymbol{\Phi}^{e}\left(x_{e+1}\right)+I_{2}^{t i p} \frac{\mathrm{d} \boldsymbol{\Phi}^{e}\left(x_{e+1}\right)^{T}}{\mathrm{~d} x} \frac{\mathrm{d} \boldsymbol{\Phi}^{e}\left(x_{e+1}\right)}{\mathrm{d} x} \\
& \boldsymbol{K}^{e}=\sum_{n l=1}\left(\int_{x_{e}}^{x_{e+1}} \int_{A^{(n l)}} z^{2} \bar{c}_{11}^{(n l)} \boldsymbol{\Psi}^{e}(x)^{T} \boldsymbol{\Psi}^{e}(x) \mathrm{d} A^{(n l)} \mathrm{d} x\right), \boldsymbol{C}^{e}=\alpha \boldsymbol{M}^{e}+\beta \boldsymbol{K}^{e} \\
& \boldsymbol{P}_{\theta}^{e}=-\sum_{n p=1}\left(\int_{x_{e}}^{x_{e+1}} \int_{A^{(n p)}} z e_{31}^{(n p)} \boldsymbol{\Psi}^{e}(x)^{T} \boldsymbol{\Omega}^{e}(z) \mathrm{d} A^{(n p)} \mathrm{d} x\right), \boldsymbol{P}_{\theta}^{e^{T}}=-\sum_{n p=1}\left(\int_{x_{e}}^{x_{e+1}} \int_{A^{(n p)}} z e_{31}^{(n p)} \boldsymbol{\Omega}^{e}(z)^{T} \boldsymbol{\Psi}^{e}(x) \mathrm{d} A^{(n p)} \mathrm{d} x\right)
\end{aligned}
$$




$$
\begin{gathered}
\boldsymbol{P}_{D}^{e}=-\sum_{n p=1}\left(\int_{x_{e}}^{x_{e+1}} \int_{A^{(n p)}} \varepsilon_{33}^{(n p)} \boldsymbol{\Omega}^{e}(z)^{T} \boldsymbol{\Omega}^{e}(z) \mathrm{d} A^{(n p)} \mathrm{d} x\right), \boldsymbol{F}^{e}=-\boldsymbol{Q}^{e} \ddot{\boldsymbol{w}}_{\text {base }}(t) \\
\boldsymbol{Q}^{(e)}=\sum_{n l=1}\left(\int_{x_{e}}^{x_{e+1}} \int_{A^{(n l)}} \rho^{(n l)} \boldsymbol{\Phi}^{e}(x)^{T} \mathrm{~d} A^{(n l)} \mathrm{d} x\right)+I_{0}^{t i p} x_{c} \frac{\mathrm{d} \boldsymbol{\Phi}^{e}\left(x_{e+1}\right)^{T}}{\mathrm{~d} x}+I_{0}^{t i p} \boldsymbol{\Phi}^{e}\left(x_{e+1}\right)^{T}
\end{gathered}
$$

It is important to note here that equation (43) consists of mass, stiffness, electromechanical coupling, and piezoelectric capacitance matrices and dynamic force vectors. These parameters are modelled at the local electromechanical element located between the end of the unimorph beam length and the tip mass. The contribution of the offset tip mass can be seen in the mass matrices and dynamic force vectors where most of the other literatures ignored this. As the unimorph-based Euler-Bernoulli beam assumption is considered, the first term from the mass matrix (rotary inertia of the unimorph) can be discarded due to the contribution of the Rayleigh beam assumption. Also, the contribution of input base excitation to the cantilever unimorph beam mathematically affects the distributed input dynamic forces into all the elements. Note that when other elements of the unimorph beam are considered in the numerical simulation, the parameter for the tip mass should be discarded from the mass matrices and input dynamic force vectors.

\subsection{Normalised global element matrices of electromechanical dynamic equations}

Constitutive electromechanical finite element dynamic equations in global matrix form reduced from the global transformation of local elements can be formulated as,

$$
\begin{aligned}
& \underset{\left(n_{m} \times n_{m}\right)}{\boldsymbol{M}} \underset{\left(n_{m} \times 1\right)}{\ddot{\boldsymbol{u}}(t)}+\underset{\left(n_{m} \times n_{m}\right)}{\boldsymbol{C}} \underset{\left(n_{m} \times 1\right)}{\dot{\boldsymbol{u}}(t)}+\underset{\left(n_{m} \times n_{m}\right)}{\boldsymbol{K}} \underset{\left(n_{m} \times 1\right)}{\boldsymbol{u}(t)}+\underset{\left(n_{m} \times n_{e}\right)}{\boldsymbol{P}} \underset{\left(n_{e} \times 1\right)}{\boldsymbol{v}(t)}=\underset{\left(n_{m} \times 1\right)}{\boldsymbol{F}} \\
& \underset{\left(n_{e} \times n_{m}\right)}{\boldsymbol{P}_{\theta}^{T}} \underset{\left.n_{m} \times 1\right)}{\dot{\boldsymbol{u}}(t)}+\underset{\left(n_{e} \times n_{e}\right)}{\boldsymbol{P}_{D}} \underset{\left(n_{e} \times 1\right)}{\dot{\boldsymbol{v}}(t)}=\boldsymbol{i}_{\left(n_{e} \times 1\right)}(t)
\end{aligned}
$$

Note that script terms $n_{m}$ and $n_{e}$ showing at each parameter in equation (45) indicate the global matrices corresponding to global displacement vectors at nodes (nodal degrees of freedom) and global electrical voltage vectors at elements (electrical degrees of freedom), respectively. For assembling the global matrices, the connectivity matrices whose discretised elements share the same node, should meet the requirements of compatibility. As the unimorph beam is a cantilevered structure under input base excitation, the fixed boundary condition at the base structure must be employed in the global matrices. For this case, the derivations for the normalised electromechanical dynamic equations and FRFs can be solved and given in the next stage. The use of MATLAB computer program not only provides the best method for solving this case but also gives the efficient computation technique. 
The solution form of time-dependent displacement can be stated in terms of the normalised modal vector and time-dependent displacement generalised coordinate as,

$$
\boldsymbol{u}(t)=\boldsymbol{\varphi}_{1} \boldsymbol{a}_{1}(t)+\boldsymbol{\varphi}_{2} \boldsymbol{a}_{2}(t)+\ldots+\boldsymbol{\varphi}_{m-1} \boldsymbol{a}_{m-1}(t)+\boldsymbol{\varphi}_{m} \boldsymbol{a}_{m}(t)=\boldsymbol{\varphi} \boldsymbol{a}(t)
$$

where the normalised modal matrix can be formulated as,

$$
\boldsymbol{\varphi}=\boldsymbol{U}\left(\boldsymbol{U}^{T} \boldsymbol{M U}\right)^{-\frac{1}{2}}
$$

It should be noted that parameters $\boldsymbol{\varphi}$ and $\boldsymbol{U}$ represent a set of the normalised modal matrix and generalised eigenvector, respectively. To obtain the eigenvector and eigenvalue solution, the undamped mechanical dynamic equation from the stiffness and mass matrices should be non-singular, since the eigenvalue equation $\left(\boldsymbol{K}-\omega^{2} \boldsymbol{M}\right) \boldsymbol{U}=0$ can be used to find the nontrivial eigenvector. Note that the normalised modal matrix is the matrix whose columns consist of the normalised eigenvectors [56]. The elemental strain field of the unimorph beam given in equation (24) can be formulated in terms of equations (24) and (46) to give,

$$
\boldsymbol{S}(x, t)=-z \boldsymbol{\Psi}^{e}(x) \boldsymbol{u}^{e}(t)=-\underset{(1 \times 4)}{z \boldsymbol{\Psi}^{e}}(x) \underset{(4 \times r)(r \times 1)}{\boldsymbol{\varphi}^{e}} a(t)=-\underset{(1 \times r)}{z \boldsymbol{\mu}^{e}}(x) \underset{(r \times 1)}{a}(t)
$$

It should be noted that the elemental strain node parameter $\boldsymbol{\mu}^{e}(x)$ based on the normalised eigenvector can be determined at certain elements and nodes of the system over the interval $x_{e} \leq x \leq x_{e+1}$. Note that collective data points for strain nodes along the unimorph beam can only be identified for each discretised element using the differential strain-displacement function $\boldsymbol{\Psi}^{e}(x)$ and elemental normalised eigenvector $\boldsymbol{\varphi}$. The normalised eigenvector at certain elements can only be determined by separating cell vector array from the normalised modal matrix in terms of the elemental degree of freedom and multimode system.

Equation (47) can be used to diagonalise the mass, stiffness and damping matrices for simplifying the computation technique. Substituting equation (46) into equation (45) and premultiplying the result by $\varphi^{T}$ gives,

$$
\begin{gathered}
\boldsymbol{\varphi}^{T} \boldsymbol{M} \boldsymbol{\varphi} \ddot{a}(t)+\boldsymbol{\varphi}^{T} \boldsymbol{C} \boldsymbol{\varphi} \dot{\boldsymbol{a}}(t)+\boldsymbol{\varphi}^{T} \boldsymbol{K} \boldsymbol{\varphi} \boldsymbol{a}(t)+\boldsymbol{\varphi}^{T} \boldsymbol{P}_{\theta} \boldsymbol{v}(t)=\boldsymbol{\varphi}^{T} \boldsymbol{F} \\
\boldsymbol{P}_{\theta}^{T} \boldsymbol{\varphi} \dot{\boldsymbol{a}}(t)+\boldsymbol{P}_{D} \dot{\boldsymbol{v}}(t)=\boldsymbol{i}_{p}(t)
\end{gathered}
$$

or simplifying equation (49) becomes,

$$
\begin{gathered}
\ddot{\boldsymbol{a}}(t)+2 \zeta \boldsymbol{\omega} \dot{\boldsymbol{a}}(t)+\boldsymbol{\omega}^{2} \boldsymbol{a}(t)+\hat{\boldsymbol{P}}_{\theta} \boldsymbol{v}(t)=-\hat{\boldsymbol{Q}}_{\ddot{\boldsymbol{w}}_{\text {base }}(t)} \\
\hat{\boldsymbol{P}}_{\theta}^{T} \dot{\boldsymbol{a}}(t)+\boldsymbol{P}_{D} \dot{\boldsymbol{v}}(t)=\boldsymbol{i}_{p}(t)
\end{gathered}
$$


Parameter $\hat{\boldsymbol{Q}} \ddot{\boldsymbol{w}}_{\text {base }}(t)$ from equation (50a) indicates nodal dynamic forces of the discretised unimorph harvester model due to the input base excitation. Other normalised parameters from equation (50) can be stated as,

$$
\begin{gathered}
\boldsymbol{\varphi}^{T} \boldsymbol{M} \boldsymbol{\varphi}=\boldsymbol{I}, \boldsymbol{\varphi}^{T} \boldsymbol{K} \boldsymbol{\varphi}=\boldsymbol{\omega}^{2}, \boldsymbol{\varphi}^{T} \boldsymbol{C} \boldsymbol{\varphi}=\alpha\left(\boldsymbol{\varphi}^{T} \boldsymbol{M} \boldsymbol{\varphi}\right)+\beta\left(\boldsymbol{\varphi}^{T} \boldsymbol{K} \boldsymbol{\varphi}\right)=\alpha \boldsymbol{I}+\beta \boldsymbol{\omega}^{2}=2 \zeta \boldsymbol{\omega} \\
\hat{\boldsymbol{P}}_{\theta}=\boldsymbol{\varphi}^{T} \boldsymbol{P}_{\theta}, \hat{\boldsymbol{P}}_{\boldsymbol{\theta}}^{T}=\boldsymbol{P}_{\theta}^{T} \boldsymbol{\varphi}, \quad \hat{\boldsymbol{Q}}=\boldsymbol{\varphi}^{T} \boldsymbol{Q}
\end{gathered}
$$

It should be noted that equation (51a) represents the orthornormality property of mechanical dynamic equations, the results of which indicate diagonal matrices.

\subsection{Normalised global scalar form of the electromechanical dynamic equations}

In simplification, the normalised global element matrices of electromechanical transverse equations from equation (50) can be reduced into scalar forms in order to formulate the multimode frequency analysis of the distributed piezoelectric component as shown in figure 2a. The electromechanical equations consist of the coupled field normalised differential equations for each elemental global coordinate component. In this case, the first form of the discretised electromechanical piezoelectric dynamic equation can be formulated for the multi-degree of freedom (multimode) system $r=1,2,3, \ldots ., N D O F$ in terms of the number of normalised piezoelectric elements $s=1,2,3, \ldots ., N E L P$ as,

$$
\ddot{a}_{r}(t)+2 \zeta_{r} \omega_{r} \dot{a}_{r}(t)+\omega_{r}^{2} a_{r}(t)+\sum_{s=1}^{N E L P} \hat{P}_{r s} v_{s}(t)=-\hat{Q}_{r} \ddot{w}_{\text {base }}(t), \quad r=1,2, \ldots, N D O F
$$

The second form of the discretised electromechanical piezoelectric dynamic equation can be formulated as,

$$
\sum_{r=1}^{N D O \hat{P}_{s r}} \dot{a}_{r}(t)+P_{D s} \dot{v}_{s}(t)=i_{P s}(t), \quad s=1,2,3, \ldots, N E L P
$$

Note that since the piezoelectric surface with the electrode layers is distributed evenly on the substructure layer, the coupled equation for the global coordinate system should be based on the electromechanical discretisation as shown in figure $2 \mathrm{~b}$. The internal parallel connection in terms of the Kirchhoff's voltage law (KVL) and Kirchhoff's current law (KCL) can be used to formulate the electrical discretised elements as,

$$
\begin{aligned}
& v_{1}(t)=v_{2}(t)=\ldots .=v_{s}(t)=v(t) \\
& i_{P 1}(t)+i_{P 2}(t)+\ldots .+i_{P S}(t)=i_{P}(t)
\end{aligned}
$$

For voltage output, the external load resistance can be used as an external circuit to connect all electrical discretised piezoelectric elements in parallel connection as, 


$$
v(t)=i_{P}(t) R_{\text {load }}
$$

Multimode FRFs of the distributed piezoelectric unimorph can be formulated. First, by modifying the first term of equation (53) algebraically corresponding with the number of the normalised piezoelectric elements. Second, employing equations (54) and (55) into the equations obtained from the first step. Third, by applying equation (54) into equation (52). Fourth, the results obtained from the second and third steps can be algebraically solved using Laplace transforms giving the result in matrix form. The superposition of equations for voltage multimode FRFs can be formulated after simplifying as,

$$
\frac{v(\mathrm{j} \omega)}{-\omega^{2} w_{\text {base }} e^{\mathrm{j} \omega t}}=\frac{\sum_{r=1}^{N D O F} \frac{\hat{Q}_{r} \mathrm{j} \omega \sum_{s=1}^{N E L P \hat{P}_{s r}}}{\omega_{r}^{2}-\omega^{2}+\mathrm{j} 2 \zeta_{r} \omega_{r} \omega}}{\sum_{s=1}^{N E L P} \mathrm{j} \omega P_{D s}-\frac{1}{R_{\text {load }}}-\sum_{r=1}^{N D O F} \frac{\mathrm{j} \omega \sum_{s=1}^{N E L P} \hat{P}_{s r} \sum_{s=1}^{N E L P} \hat{P}_{r s}}{\omega_{r}^{2}-\omega^{2}+\mathrm{j} 2 \zeta_{r} \omega_{r} \omega}}
$$

The multimode FRF of the electric current output related to the input base transverse acceleration can be stated as,

$$
\frac{i_{p}(\mathrm{j} \omega)}{-\omega^{2} w_{\text {base }} e^{\mathrm{j} \omega t}}=\frac{\frac{1}{R_{\text {load }}} \sum_{r=1}^{N D O F} \frac{\hat{Q}_{r} \mathrm{j} \omega \sum_{s=1}^{N E L P} \hat{P}_{s r}}{\omega_{r}^{2}-\omega^{2}+\mathrm{j} 2 \zeta_{r} \omega_{r} \omega}}{\sum_{s=1}^{N E L P} \mathrm{j} \omega P_{D s}-\frac{1}{R_{\text {load }}}-\sum_{r=1}^{N D O F} \frac{\mathrm{j} \omega \sum_{s=1}^{N E L P} \hat{P}_{s r} \sum_{s=1}^{N E L P} \hat{P}_{r s}}{\omega_{r}^{2}-\omega^{2}+\mathrm{j} 2 \zeta_{r} \omega_{r} \omega}}
$$

The power harvesting multimode FRF related to the input transverse acceleration can be formulated as,

$$
\frac{P_{p}(\mathrm{j} \omega)}{\left(-\omega^{2} w_{\text {base }} e^{\mathrm{j} \omega t}\right)^{2}}=\left[\frac{\frac{1}{\sqrt{R_{\text {load }}}} \sum_{r=1}^{N D O F} \frac{\hat{Q}_{r} \mathrm{j} \omega \sum_{s=1}^{N E L P} \hat{P}_{s r}}{\omega_{r}^{2}-\omega^{2}+\mathrm{j} 2 \zeta_{r} \omega_{r} \omega}}{\sum_{s=1}^{N E L P} \mathrm{j} \omega P_{D s}-\frac{1}{R_{\text {load }}}-\sum_{r=1}^{N E L P} \frac{\mathrm{j} \omega \sum_{s=1}^{N E L} \hat{P}_{s r} \sum_{s=1}^{N E L P} \hat{P}_{r s}}{\omega_{r}^{2}-\omega^{2}+\mathrm{j} 2 \zeta_{r} \omega_{r} \omega}}\right]^{2}
$$

To formulate the optimal multimode FRF of power harvesting, the reduced optimal load resistance needs to be formulated first. For this case, equation (58) can be differentiated with respect to load resistance and the differentiable power function can be set to zero to give the optimal load resistance as, 


$$
R_{\text {load }}^{\text {opt }}=\frac{\sqrt{X(\omega)^{2}+Y(\omega)^{2}}}{X(\omega)^{2}+Y(\omega)^{2}}
$$

where

$$
X(\omega)=\sum_{s=1}^{N E L P} \omega P_{D s}-\sum_{r=1}^{N D O F} \frac{\omega \sum_{s=1}^{N E L P} \hat{P}_{s r} \sum_{s=1}^{N E L P} \hat{P}_{r s}\left(\omega_{r}{ }^{2}-\omega^{2}\right)}{\left(\omega_{r}{ }^{2}-\omega^{2}\right)^{2}+\left(2 \zeta_{r} \omega_{r} \omega\right)^{2}}, \quad Y(\omega)=\sum_{r=1}^{N D O F} \frac{\omega \sum_{s=1}^{N E L P} \hat{P}_{s r} \sum_{s=1}^{N E L P} \hat{P}_{r s}\left(2 \zeta_{r} \omega_{r} \omega\right)}{\left(\omega_{r}{ }^{2}-\omega^{2}\right)^{2}+\left(2 \zeta_{r} \omega_{r} \omega\right)^{2}}
$$

It should be noted that the optimal load resistance can be substituted back into equation (58) to give the optimal power harvesting. Moreover, the multimode FRF representing the transverse displacement relative to the input transverse acceleration can be obtained as,

$$
\frac{a_{r}(\mathrm{j} \omega)}{-\omega^{2} w_{\text {base }} e^{\mathrm{j} \omega t}}=-\frac{1}{\omega_{r}^{2}-\omega^{2}+\mathrm{j} 2 \zeta_{r} \omega_{r} \omega}\left(\frac{\left(\sum_{s=1}^{N E L P} \mathrm{j} \omega P_{D s}+R_{L}\right) \hat{Q}_{r}}{\sum_{s=1}^{N E L P} \mathrm{j} \omega P_{D s}-\frac{1}{R_{\text {load }}}-\sum_{r=1}^{N D O F} \frac{\mathrm{j} \omega \sum_{s=1}^{N E L P} \hat{P}_{s r} \sum_{s=1}^{N E L P} \hat{P}_{r s}}{\omega_{r}^{2}-\omega^{2}+\mathrm{j} 2 \zeta_{r} \omega_{r} \omega}}\right)
$$

Before equation (61) is modified into the transverse displacement FRF with respect to the input transverse acceleration at any position along the unimorph beam $(x)$, the characteristic transverse motion of the unimorph beam can be reformulated in terms of the natural normal mode multiplied by the time-dependent transverse displacement using equations (21) and (46) to give,

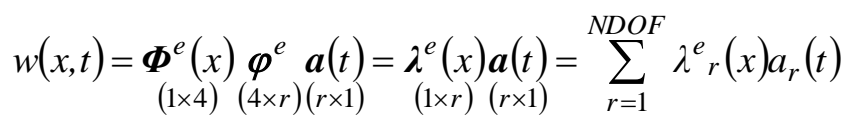

Moreover, the characteristic motions for tip mass offset over the interval $L \leq x \leq L+L_{t i p}$ can be formulated using the characteristic motions of the tip of the beam. Note that, as mentioned previously; since the tip mass offset is assumed to undergo rigid body motion, its motion solely depends upon the tip of the beam's transverse rectilinear and rotation or slope motions. In this point, the transverse displacement response of the tip mass offset can be formulated as,

$$
\begin{aligned}
& \boldsymbol{w}\left(L+L_{t i p}, t\right)=\boldsymbol{w}(L, t)+L_{t i p} \frac{\mathrm{d} \boldsymbol{w}(L, t)}{\mathrm{d} x}=\left(\underset{(1 \times 4)}{\boldsymbol{\Phi}^{e}(L)+L_{t i p}} \frac{\mathrm{d} \boldsymbol{\Phi}^{e}(L)}{\underset{(1 \times 4)}{\mathrm{d} x}}\right) \underset{(4 \times r)(r \times 1)}{\boldsymbol{\varphi}^{e}} \underset{(t)}{\boldsymbol{a}(t)}
\end{aligned}
$$

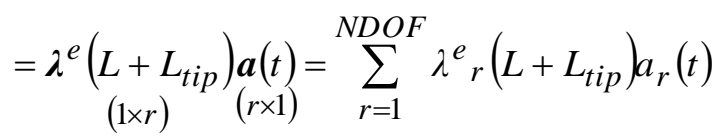

Corresponding to equations (61) and (62), the FRF that relates the multimode transverse displacement to the input base acceleration of the unimorph beam can be formulated as, 


$$
\frac{w(x, \mathrm{j} \omega)}{-\omega^{2} w_{\text {base }} e^{\mathrm{j} \omega t}}=-\sum_{r=1}^{N D O F}\left[\frac{\lambda^{e}{ }_{r}(x)}{\omega_{r}^{2}-\omega^{2}+\mathrm{j} 2 \zeta_{r} \omega_{r} \omega}\left(\frac{\left(\sum_{s=1}^{N E L P} \mathrm{j} \omega P_{D s}+R_{L}\right) \hat{Q}_{r}}{\sum_{s=1}^{N E L P} \mathrm{j} \omega P_{D s}-\frac{1}{R_{\text {load }}}-\sum_{r=1}^{N D O F} \frac{\mathrm{j} \omega \sum_{s=1}^{N E L P} \hat{P}_{s r} \sum_{s=1}^{N E L P} \hat{P}_{r s}}{\omega_{r}^{2}-\omega^{2}+\mathrm{j} 2 \zeta_{r} \omega_{r} \omega}}\right)\right]
$$

For tip mass offset, the multimode transverse displacement FRFs can also be formulated in terms of equations (61) and (63) to give,

$$
\frac{w\left(L+L_{\text {tip }}, \mathrm{j} \omega\right)}{-\omega^{2} w_{\text {base }} e^{\mathrm{j} \omega t}}=-\sum_{r=1}^{N D O F}\left[\frac{\lambda^{e}{ }_{r}\left(L+L_{\text {tip }}\right)}{\omega_{r}{ }^{2}-\omega^{2}+\mathrm{j} 2 \zeta_{r} \omega_{r} \omega}\left(\frac{\left(\sum_{s=1}^{N E L P} \mathrm{j} \omega P_{D s}+R_{L}\right) \hat{Q}_{r}}{\sum_{s=1}^{N E L P} \mathrm{j} \omega P_{D s}-\frac{1}{R_{\text {load }}}-\sum_{r=1}^{N D O F} \frac{\mathrm{j} \omega \sum_{s=1}^{N E L P} \hat{P}_{S r} \sum_{s=1}^{N E L P} \hat{P}_{r s}}{\omega_{r}{ }^{2}-\omega^{2}+\mathrm{j} 2 \zeta_{r} \omega_{r} \omega}}\right)\right]
$$

For experimental study, a Laser Doppler Vibrometer (LDV) was used for capturing dynamic responses from the small beam and tip mass structures under input base motion to give the absolute motions. For this case, equations (64) and (65) need to be modified in terms of the absolute motion. Since the combination between the base and relative motions defines the absolute motion, the multimode FRFs of absolute transverse displacement and velocity at any position along the unimorph beam and the tip mass offset can be formulated as,

$$
\begin{aligned}
& \frac{w_{a b s}(x, \mathrm{j} \omega)}{-\omega^{2} w_{b a s} e^{\mathrm{j} \omega t}}=\frac{w_{\text {bas }} e^{\mathrm{j} \omega t}+w(x, \mathrm{j} \omega)}{-\omega^{2} w_{\text {bas }} e^{\mathrm{j} \omega t}}=-\frac{1}{\omega^{2}}+\frac{w(x, \mathrm{j} \omega)}{-\omega^{2} w_{\text {base }} e^{\mathrm{j} \omega t}} \\
& \frac{\dot{w}_{a b s}(x, \mathrm{j} \omega)}{-\omega^{2} w_{b a s} e^{\mathrm{j} \omega t}}=\frac{\frac{\mathrm{d}}{\mathrm{d} t}\left[w_{\text {base }} e^{\mathrm{j} \omega t}+w(x, \mathrm{j} \omega)\right]}{-\omega^{2} w_{b a s} e^{\mathrm{j} \omega t}}=\frac{1}{\mathrm{j} \omega}+\mathrm{j} \omega \frac{w(x, \mathrm{j} \omega)}{-\omega^{2} w_{b a s} e^{\mathrm{j} \omega t}} \\
& \frac{w_{a b s}\left(L+L_{t i p}, \mathrm{j} \omega\right)}{-\omega^{2} w_{\text {base }} e^{\mathrm{j} \omega t}}=\frac{w_{\text {base }} e^{\mathrm{j} \omega t}+w\left(L+L_{t i p}, \mathrm{j} \omega\right)}{-\omega^{2} w_{\text {bas }} e^{\mathrm{j} \omega t}}=-\frac{1}{\omega^{2}}+\frac{w\left(L+L_{t i p}, \mathrm{j} \omega\right)}{-\omega^{2} w_{\text {base }} e^{\mathrm{j} \omega t}} \\
& \frac{\dot{w}_{a b s}\left(L+L_{t i p}, \mathrm{j} \omega\right)}{-\omega^{2} w_{b a s} e^{\mathrm{j} \omega t}}=\frac{\frac{\mathrm{d}}{\mathrm{d} t}\left[w_{b a s} e^{\mathrm{j} \omega t}+w\left(L+L_{t i p}, \mathrm{j} \omega\right)\right]}{-\omega^{2} w_{b a s} e^{\mathrm{j} \omega t}}=\frac{1}{\mathrm{j} \omega}+\mathrm{j} \omega \frac{w\left(L+L_{t i p}, \mathrm{j} \omega\right)}{-\omega^{2} w_{b a s} e^{\mathrm{j} \omega t}}
\end{aligned}
$$

\section{Experimental validation: results and discussion}

In this section, the multimode FRFs for the velocity, electrical voltage, current, and power outputs using the new electromechanical finite element equations are investigated and validated with the 
experimental studies with the variable load resistance (resistive shunt circuit). The input base transverse acceleration onto unimorph structures was chosen to be $1 \mathrm{~m} / \mathrm{s}^{2}$. The piezoelectric unimorph structure was made from the PZT PSI-5A4E material (Piezo Systems Inc., Woburn, MA) and the rigid body of the offset tip mass was subsequently attached. The geometrical structures of the piezoelectric unimorph with tip mass offset can be seen in figure 4 and detail of the tip mass geometry including formulas can be seen in appendix D. Note that since the tip mass was glued on the top surface of the substructure, the extra substructure length $l_{t}$ was assumed to be a rigid body contributing the tip mass offset. The complete properties of the unimorph are given in table 1 . In figure 4 , the unimorph length $L$ and width $b$ with piezoelectric thickness $h_{p}$ and substructure (brass) thickness $h_{s}$ were set to $60 \mathrm{~mm}$, $6 \mathrm{~mm}, 0.127 \mathrm{~mm}$ and $0.5 \mathrm{~mm}$, respectively. The tip mass configurations were calculated according to the geometry and material property. The dimensions of tip mass offset with $l_{t}, h_{t}$ and $b$ (width) were set to $15 \mathrm{~mm}, 10 \mathrm{~mm}$ and $6 \mathrm{~mm}$, respectively.

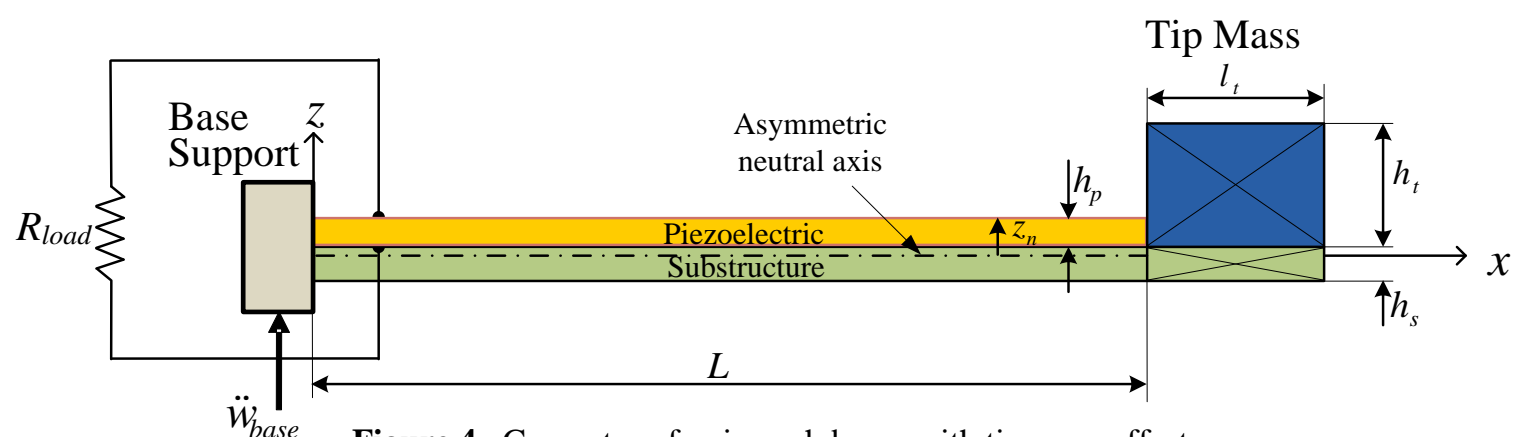

Figure 4. Geometry of unimorph beam with tip mass offset.

Table 1. Properties of the piezoelectric unimorph system.

\begin{tabular}{lll}
\hline Material properties & Piezoelectric & Brass \\
\hline Young's modulus, $\bar{c}_{11}(\mathrm{GPa})$ & 66 & 105 \\
Density, $\rho\left(\mathrm{kg} / \mathrm{m}^{3}\right)$ & 7800 & 9000 \\
Piezoelectric constant, $d_{31}(\mathrm{pm} / \mathrm{V})$ & -190 & - \\
Permittivity, $\varepsilon_{33}^{T}(\mathrm{~F} / \mathrm{m})$ & $1800 \varepsilon_{\mathrm{o}}$ & - \\
permittivity of free space, $\varepsilon_{\mathrm{O}}(\mathrm{pF} / \mathrm{m})$ & 8.854 & - \\
\hline
\end{tabular}

As shown from the experimental setup in figure 5, the cantilevered piezoelectric unimorph beam with the tip mass offset was clamped at the base structure under input dynamic excitation. The input acceleration from the base structure was measured using the B \& $\mathrm{K}$ accelerometer. The B \& $\mathrm{K}$ exciter connected to the power amplifier was used as the input dynamic excitation. The wave function generator connected to the power amplifier was used to control the input excitation using the sine sweep from the frequencies of $5 \mathrm{~Hz}$ to $1000 \mathrm{~Hz}$ with frequency increment of $0.3125 \mathrm{~Hz}$ and 40 averages controlled from the B \& K FFT Pulse analyser software in order to obtain very smooth 
frequency response and to avoid aliasing components. Dynamic velocity of the tip mass offset was measured using a Polytec laser vibrometer with a measurement range full scale of $500 \mathrm{~mm} / \mathrm{s}$ and low pass filter setting of $22 \mathrm{KHz}$. Before operating the laser, a small reflecting film was attached on the top of the tip mass. Moreover, all signal measurements from the accelerometer, piezoelectric unimorph and vibrometer were connected to the sophisticated B \& K FFT Analyzer 3560B data acquisition device with five channels with maximum frequency span up to $25.6 \mathrm{kHz}$. All processing signals through the analyzer displayed the measurement results using the FFT pulse software. Note that all sensitivities for scaling factors given from B \& $\mathrm{K}$ accelerometer of $9.633 \mathrm{mV} / \mathrm{ms}^{-2}$ and laser vibrometer of $125 \mathrm{~mm} / \mathrm{s} . \mathrm{V}\left(8 \mathrm{~V} / \mathrm{m}^{-1} \mathrm{~s}^{-1}\right)$ were inserted into the FFT pulse in order to obtain the correct results. Since the acceleration is given in per-unit acceleration in $\mathrm{m} / \mathrm{s}^{2}$, all results of FRFs given from the experiment and the numerical analysis in the next stage are given in per- unit acceleration in $\mathrm{m} / \mathrm{s}^{2}$. Note that the B \& K pulse software also provides facility (unit organiser) for modifying the units of amplitude of FRF, for example; the amplitudes from the laser measurement can be stated into the velocity or displacement per-unit acceleration.

The first four modes of the unimorph beam as shown in figure 6 can be used to identify the smoothness of mode shapes using the different sizes of a number of discretised elements. Although the resonant frequencies with discretisation using lower number of elements seem to be quite close to each other, the mode shapes seem to be quite coarse. These results show that the proposed 50 electromechanical discretised elements appear to be sufficient to provide accurate results that can give smooth mode shapes including the natural frequencies. With the 50 electromechanical discretised elements, the strain node along unimorph beam as shown in figure 7 can be determined using the inflection point of the second order differential shape function of the strain-displacement relationship as shown in equation (48). As the surfaces of the piezoelectric material are covered with the electrode layers, the inflection point can be used to identify the location of strain nodes along the beam indicating the change of strains (tension and compressive strains) between two segments of the piezoelectric structure. This can avoid the reduction of the power output at the second or higher modes. Further detail of the parametric strain node cases associated with the experimental study in terms of electrical connection patterns can be discussed in future investigations. For this case, our main purpose of this numerical study is to present the new electromechanical finite element vibration techniques and show the validation with experimental studies. 

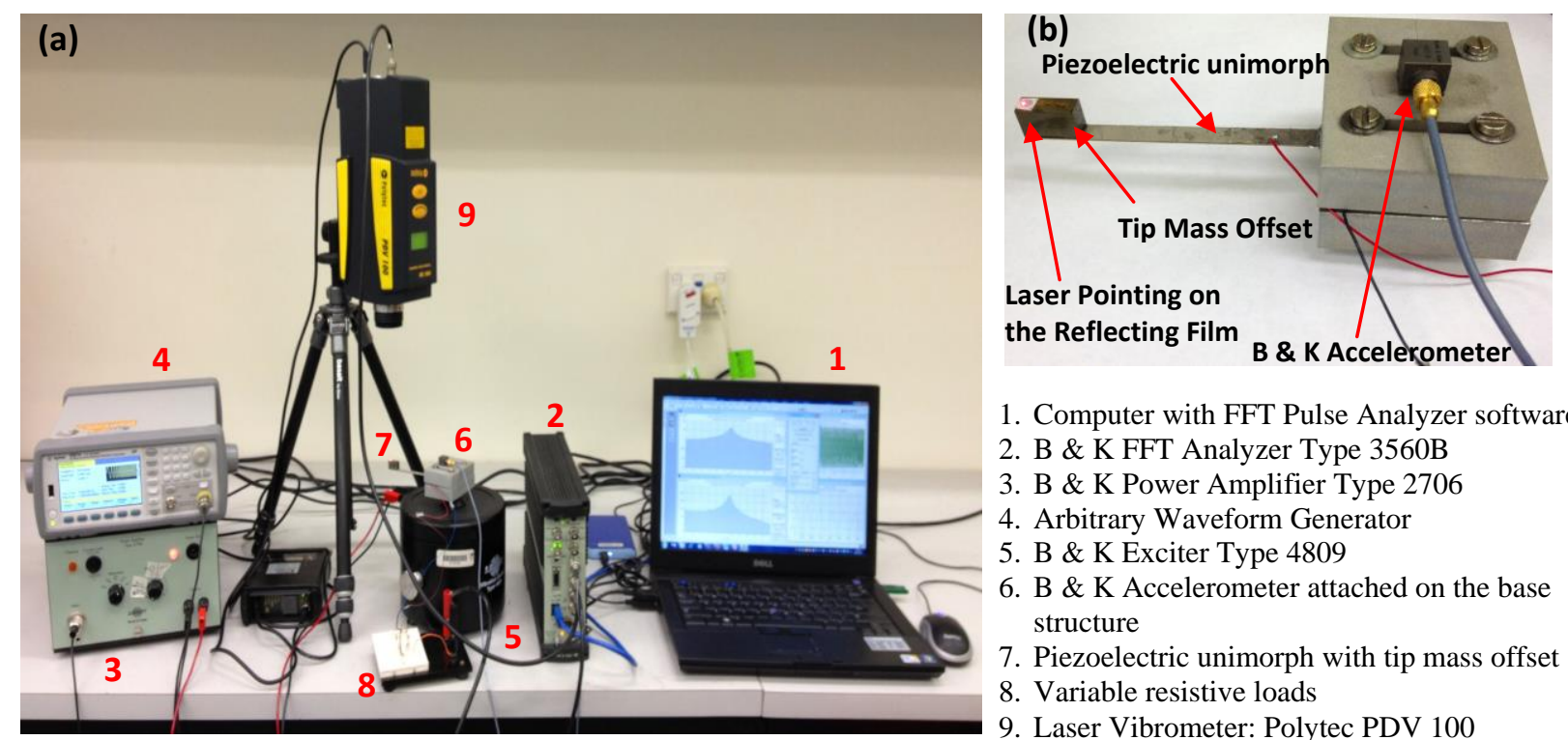

1. Computer with FFT Pulse Analyzer software

2. B \& K FFT Analyzer Type 3560B

3. B \& K Power Amplifier Type 2706

4. Arbitrary Waveform Generator

5. B \& K Exciter Type 4809

6. B \& K Accelerometer attached on the base structure

7. Piezoelectric unimorph with tip mass offset

8. Variable resistive loads

9. Laser Vibrometer: Polytec PDV 100

Figure 5. (a) Experimental setup and (b) piezoelectric unimorph beam with tip mass offset clamped on the base structure.
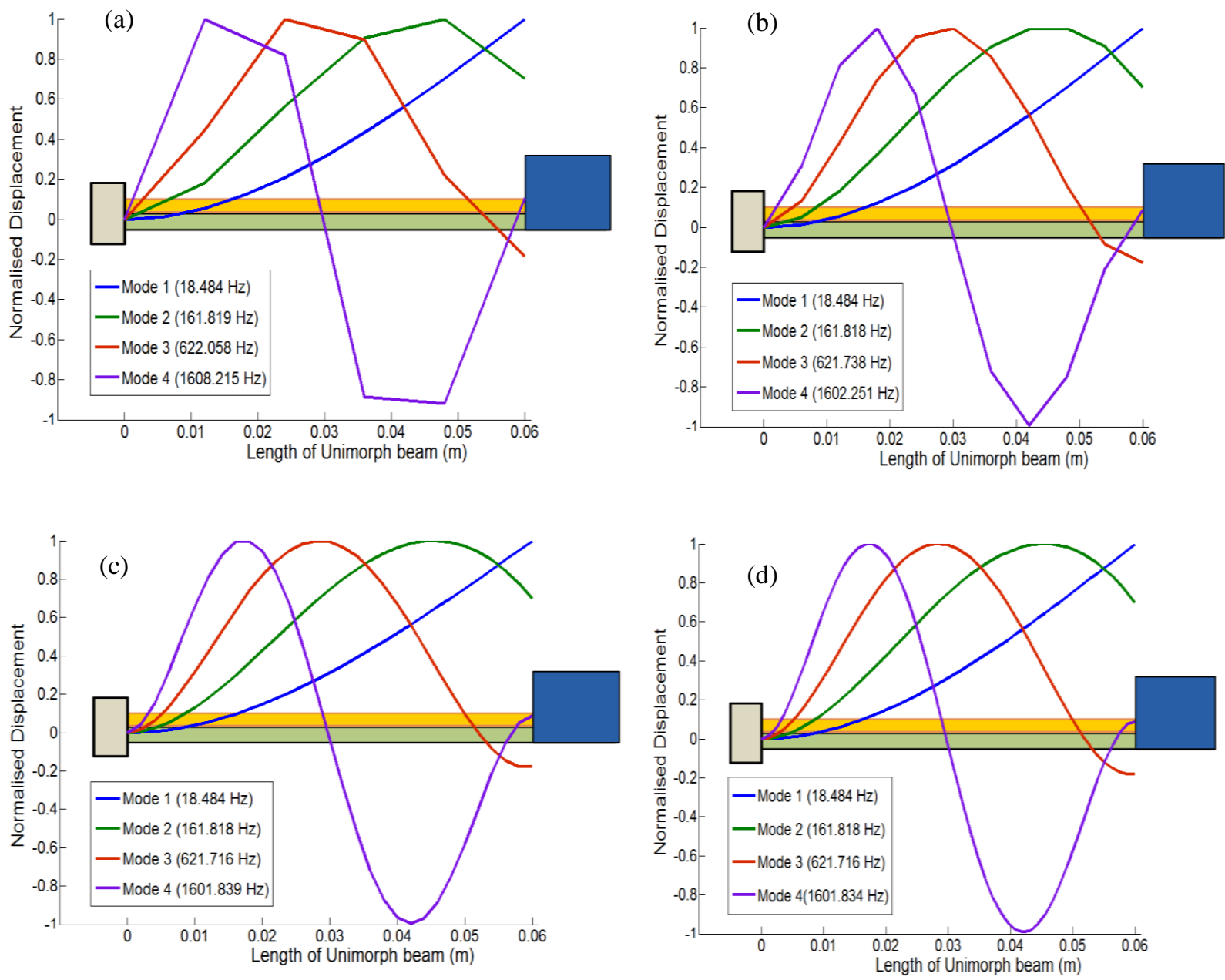

Figure 6. Normalised displacement mode shape with (a) 5 elements, (b) 10 elements, (c) 30 elements and (d) 50 elements. 


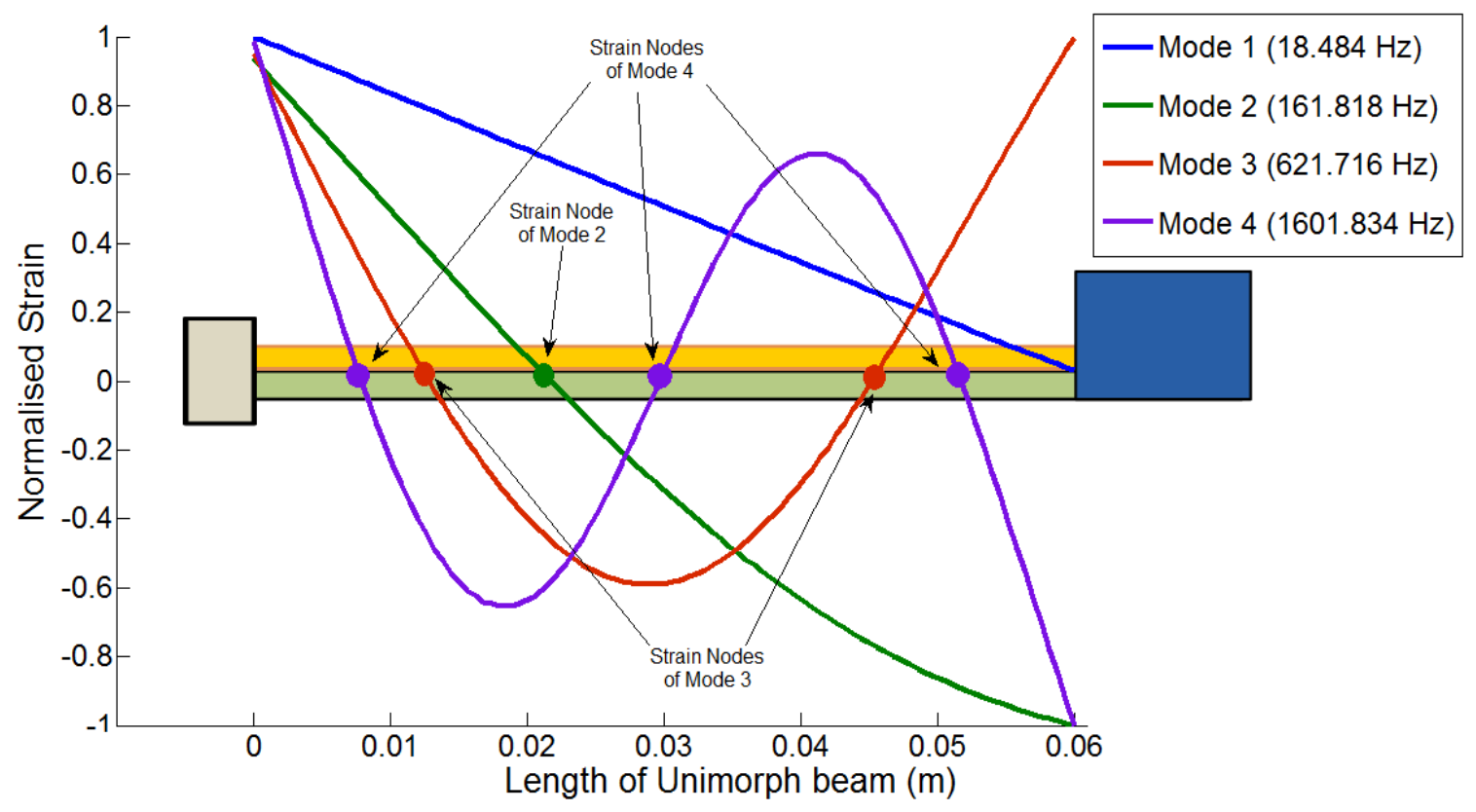

Figure 7. Normalised strain mode shape with 50 Elements.

In figure 8(a), the absolute tip velocity FRFs and experimental results for the first mode show very good agreement under the variable load resistance. As can be seen, the higher amplitudes can be achieved when load resistances and frequencies shift from the short to open circuits. By viewing figure $8(\mathrm{~b})$, the maximum tip absolute velocity responses at the short and open circuit resonance frequencies of $18.5 \mathrm{~Hz}$ and $18.9 \mathrm{~Hz}$ were reached for load resistances approaching the lower and the higher values (from short to open circuit load resistances), respectively. This behaviour can also be seen more obviously in figure 8(c). In many cases of vibration, the maximum dynamic displacement is generally avoided for most structures. It should be noted here that the power harvesting response can be shown to give the best results without having the maximum displacement as further discussed next part. Note that the resonance frequency and amplitude response can shift due to resistive shunt damping from the variable load resistance where this affects the electromechanical behaviour of the piezoelectric element. In addition, the physical behaviour of the piezoelectric unimorph, involving piezoelectric couplings and internal capacitance, also adds electromechanical damping as well as electromechanical stiffness. At this point, it is realised that the damping effects consist of the mechanical and electrical components due to the electromechanical behaviour of the dimensional structure, external load resistance and material properties of the piezoelectric and substructure. Identification of mechanical damping with very low load resistance can be obtained by matching the amplitude of the experimental and theoretical tip absolute velocity and voltage FRFs. Mechanical damping ratio of the first mode $\zeta_{1}=0.0162$ under a very low load resistance of $562 \Omega$ was obtained (approaching the short circuit resistance) because using the actual short circuit load resistance $\left(R_{\text {load }}=\right.$ 
0) will result in the theoretical voltage FRF to be zero where the tip absolute displacement cannot be identified. This situation cannot be used to identify the voltage and displacement FRF behaviours of the electromechanical system.

With variation in the load resistance, the first mode of the voltage FRFs also varies while increasing load resistance as shown in figure 9(a). For this case, the resonance frequency shifts to the higher value from short to open circuit load resistances followed by an increase in the amplitude. Note that when the voltage FRF with load resistance is close to open circuit, the amplitude remains constant.
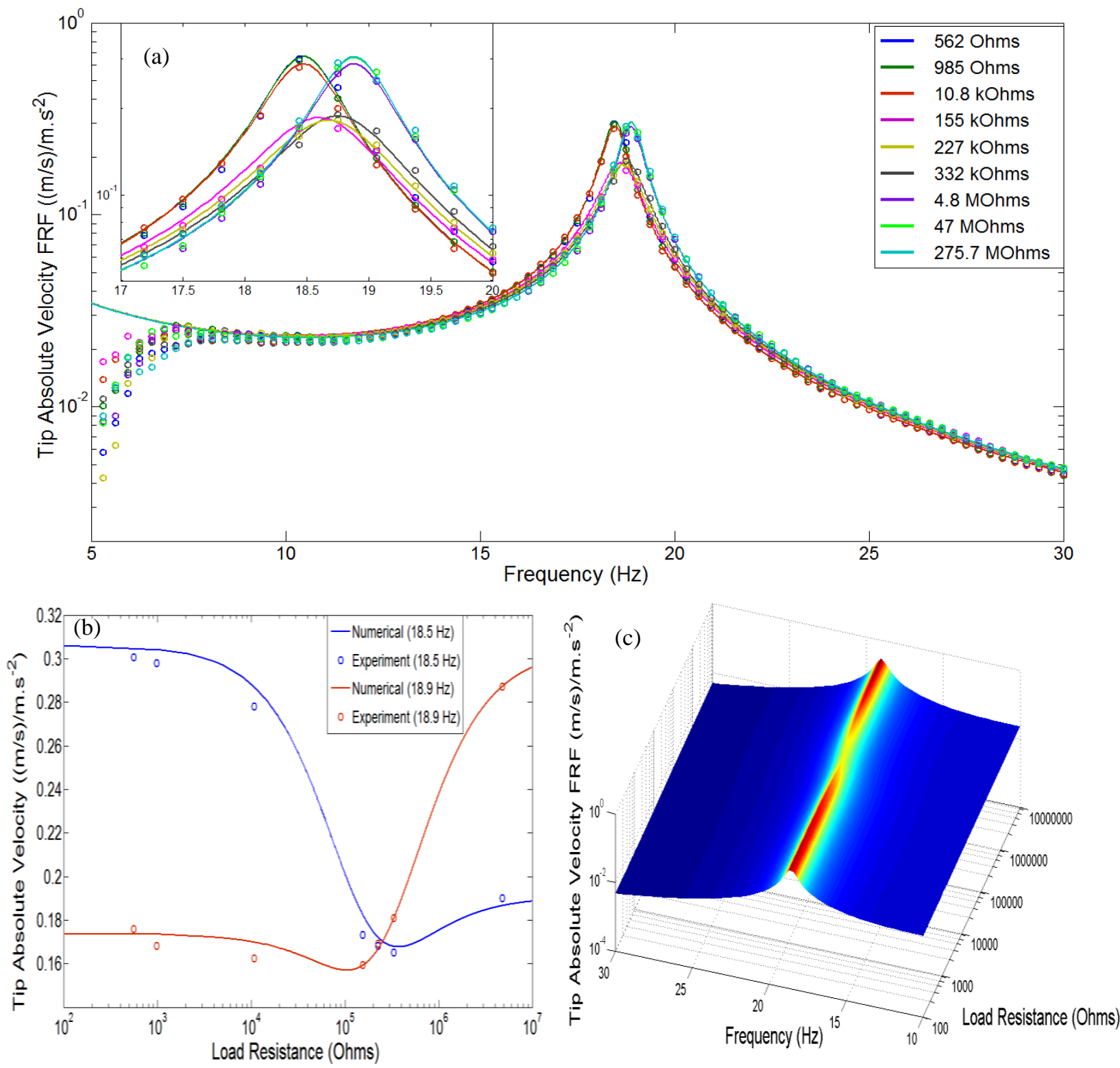

Figure 8. Tip absolute velocity FRF with (a) numerical (solid lines) and experimental results (round dot),

(b) variable load resistances under the short circuit and open circuit resonance frequencies and

(c) three dimensional analysis. 
Figure 9(b) shows the trend of voltage behaviour under variable load resistance giving good agreement between numerical and experimental results. In general form, the voltage under short and open circuit resonances indicates a slight increase with increasing load resistance. However, the maximum open circuit resonance amplitude gave a higher value compared with the short circuit resonance when load resistance passed over the transitional or critical point of the short and open circuit amplitudes. Note that the critical point of the amplitude response between short and open circuit resonance frequencies shows the same amplitude. The change of frequency due to the variable load resistance can also be seen more obviously in figure $9(\mathrm{c})$. For electrical current FRFs as shown in
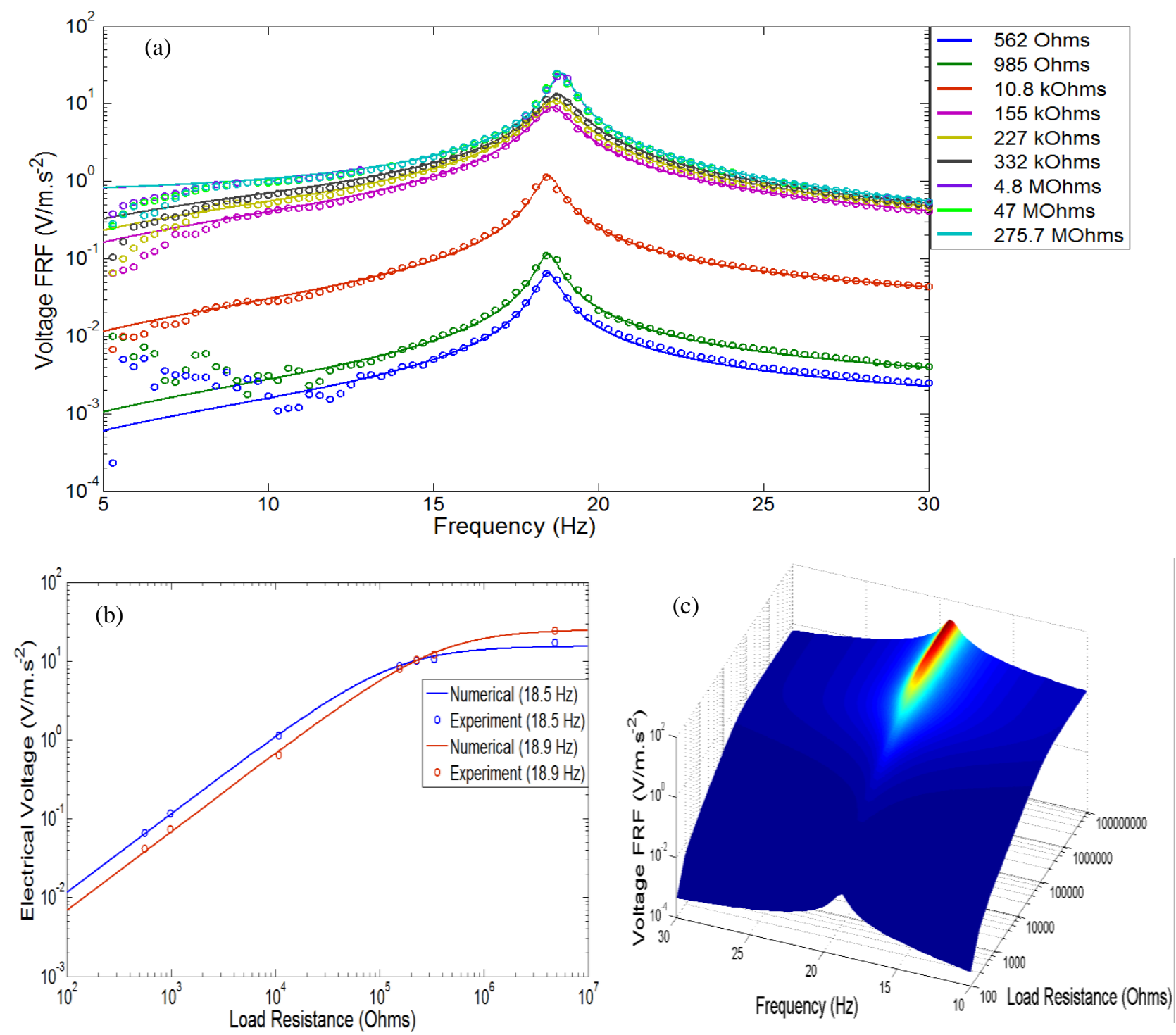

Figure 9. Voltage FRF with (a) numerical (solid lines) and experimental results (round dot),

(b) variable load resistances under the short circuit and open circuit resonance frequencies and

(c) three dimensional analysis. 
figure 10(a), the trend shows opposite behaviour to that of the voltage FRFs. The electrical current shows the highest amplitude when the load resistance approaches to the open circuit. Again, in the opposite trend with the voltage response, the variation of electric current with variable load resistance under short and open circuit resonance frequencies as shown in 10(b) reaches the maximum amplitude with decreasing load resistance. This trend can be seen more obviously in the three dimensional current FRF given in figure 10(c). Another important aspect related to the FRFs can also be considered to be the relationships between the dynamic velocity, electrical voltage and current. The absolute velocity amplitude with the load resistance approaching short circuit seems to increase, where the voltage amplitude decreases with the increasing electrical current. Conversely, the velocity amplitude with the load resistance approaching open circuit tends to increase where the voltage amplitude increases with the decreasing electrical current.
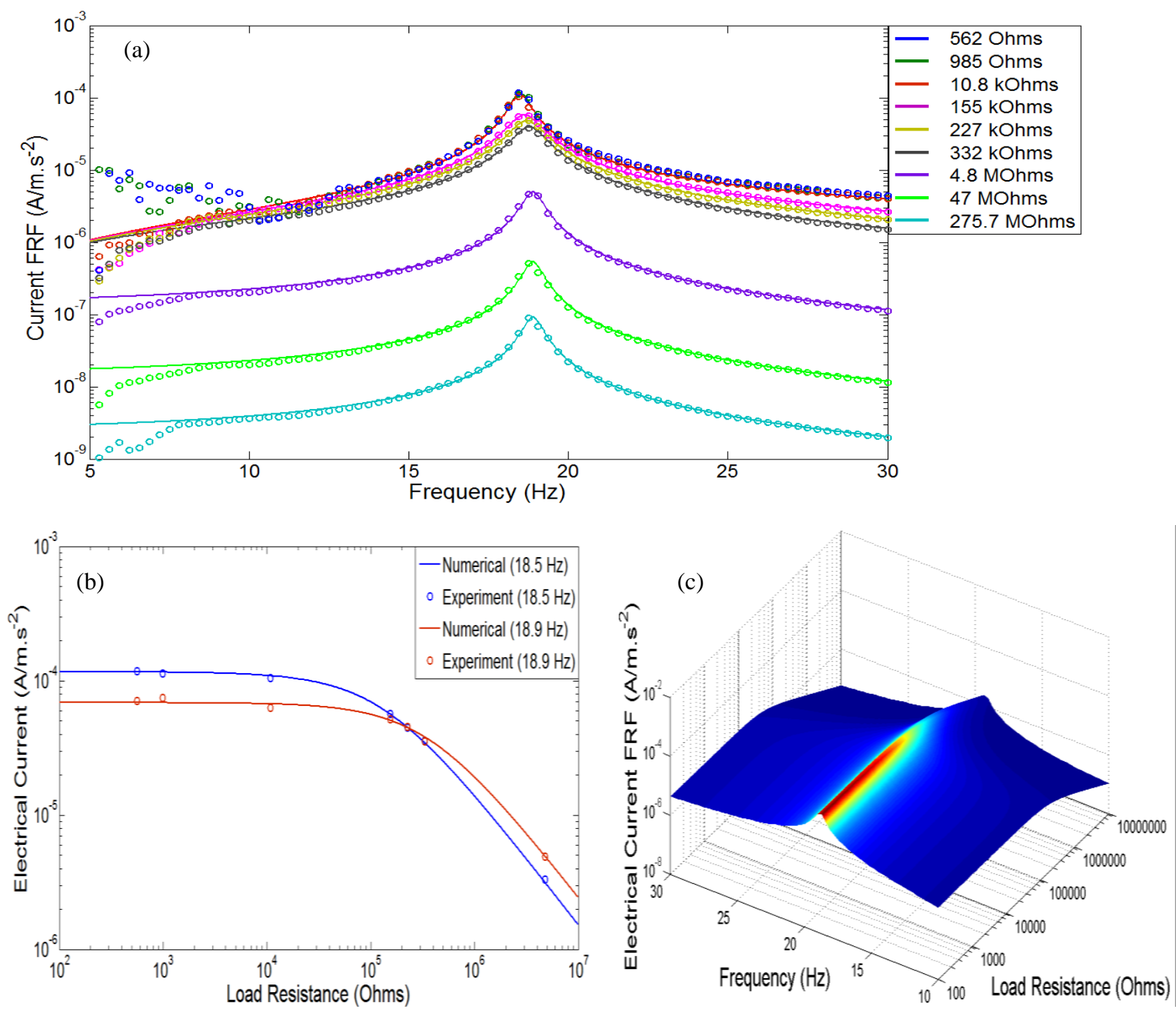

Figure 10. Electrical current FRF with (a) numerical (solid lines) and experimental results (round dot),

(b) variable load resistances under the short circuit and open circuit resonance frequencies and

(c) three dimensional analysis. 
However, the power harvester FRFs especially near the first mode response as shown in figure 11(a) seems to behave with different trend where the short and open circuit load resistances tend to decrease the amplitude. It can be said that the highest velocity amplitudes at the short and open circuit load resistances do not show the highest power amplitudes. In different configuration with velocity, voltage and current, the variation of power harvesting under short and open circuit resonance frequencies as shown figure 11(b) show two different optimum load resistances, giving the optimum amplitudes. In the three dimensional graph as shown in figure 11(c), the optimum power harvesting amplitudes can also be seen clearly at the regions away from the short and open circuit load resistances.
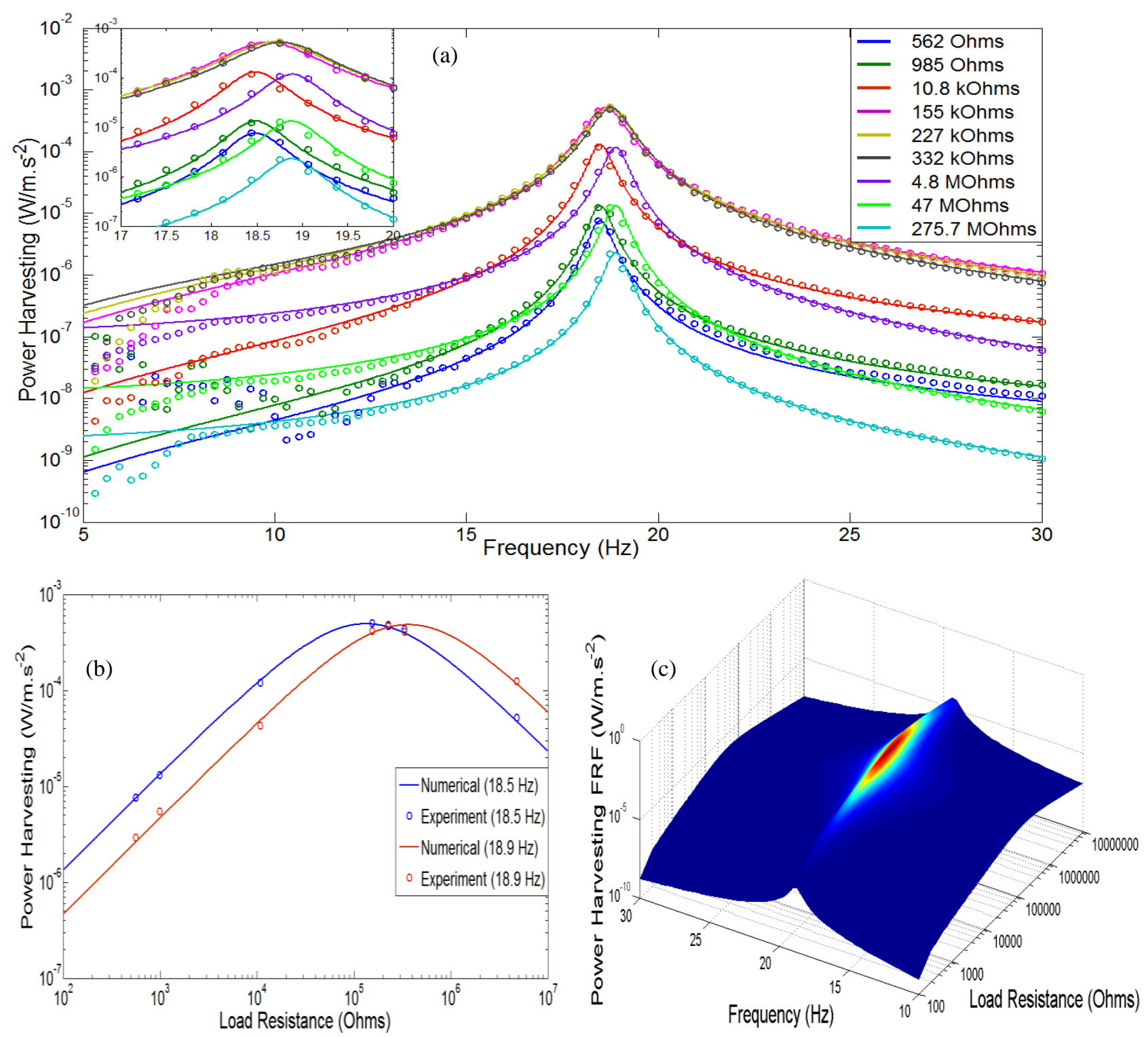

Figure 11. Power FRF with (a) numerical (solid lines) and experimental results (round dot), (b) variable load resistances under the short circuit and open circuit resonance frequencies and (c) three dimensional analysis. 

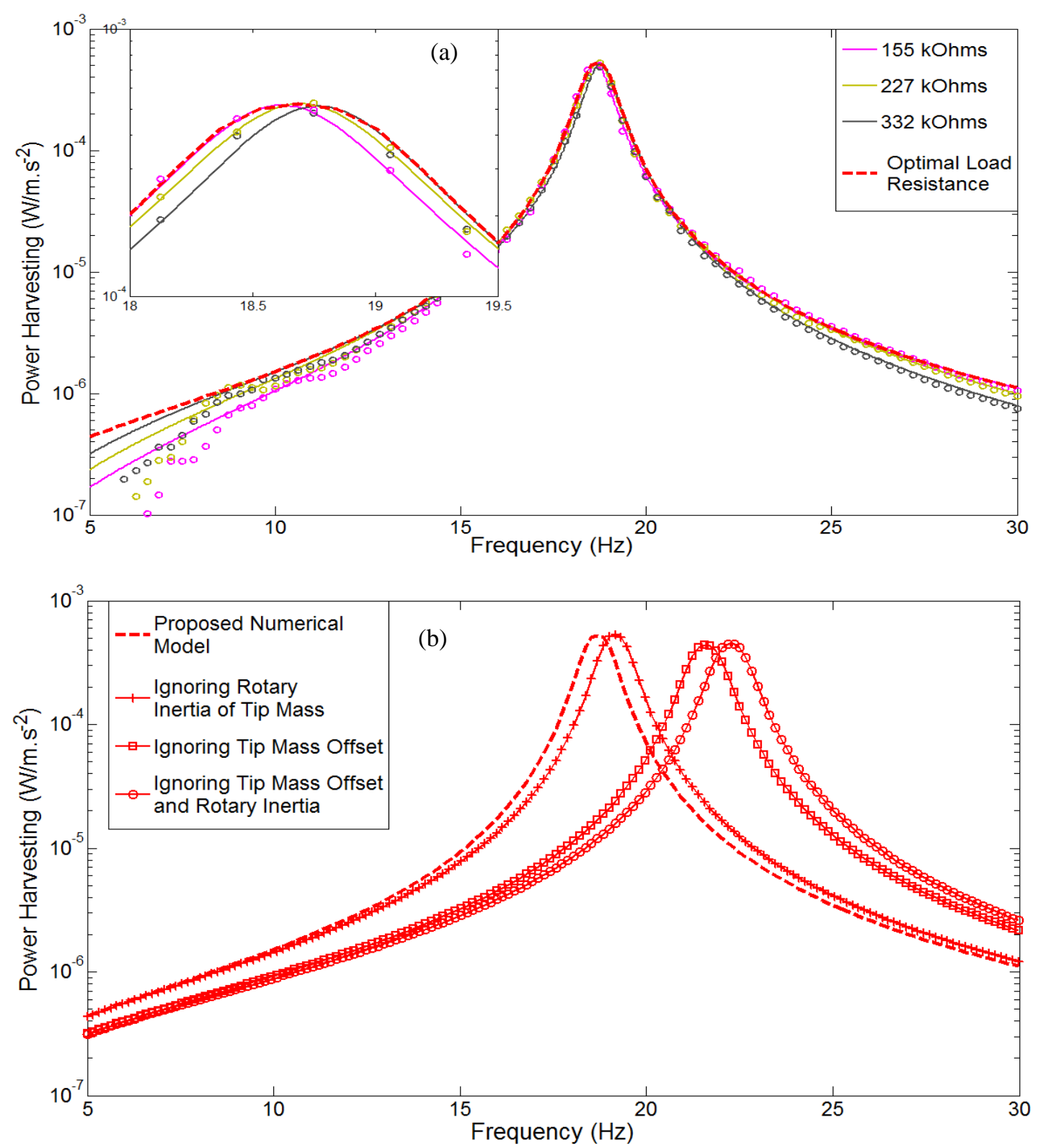

Figure 12. Identification of the optimal power FRF with (a) numerical (dash lines) and experimental results (round dot) based on the optimal load resistance and related to other load resistances and (b) different cases of tip mass offset.

The optimal power harvesting based on the optimal load resistance can also be seen in figure 12a where the absolute maximum power amplitude point coincides with the load resistance of $227 \mathrm{k} \Omega$ in the resonance frequency of $18.70 \mathrm{~Hz}$. However, the optimal load resistance in the off-resonance regions seem to approach and overlap with the load resistances of $155 \mathrm{k} \Omega$ and $332 \mathrm{k} \Omega$ where the experimental results also are very close to these off-resonance regions except for the driving frequency below $8 \mathrm{~Hz}$ due to unwanted noise. By scrutinising the optimal power harvesting response, the numerical modelling result shown in figure $12 \mathrm{~b}$ shows that ignoring the rotary inertia of the tip mass results in the shift of the resonance frequency to a higher value. The resonance difference can 
also be seen to be more pronounced if the model ignores the offset distance of the tip mass measured from its centroid to the end of the unimorph beam length. Neglecting both the offset and rotary inertia of the tip mass results in a larger modelling error. It can be seen that when designing the power harvesting device either micro- or -meso scale, the mathematical model should include the effect of the offset and rotary inertia of the tip mass.

\section{Conclusion}

The new numerical study proposed in this paper addresses five important points.

- The electromechanical discretisation was numerically developed, since the actual piezoelectric material covered by the thin electrode layers only generates a single voltage across the electrical load. The electromechanical discretisation introduced here consists of the mechanical discretised elements for elemental displacement fields and the parallel electrical discretised elements for multi-output electrical current. The previous published papers related to the piezoelectric finite element have not considered this in their mathematical modelling, although many of them showed different applications.

- Instead of developing the kinematic equations of the unimorph beam, the tip mass offset was formulated, since it contributes significantly into the mass matrices and input dynamic force vector. The benefit of locating the tip mass with its centroid away from the end of the piezoelectric beam is for modelling various applications especially MEMS power harvesters. The improved designs avoid contact between the brittle piezoelectric material and the tip mass. The previous published papers related to MEMS have ignored this in their mathematical modelling.

- The numerical techniques provide physical interrelationship between electrode effect, coupled field equations, kinematic equations, electromechanical discretisation, and electromechanical dynamic equations.

- The reduced constitutive electromechanical finite element equations were further derived into global normalised scalar form of the electromechanical dynamic equations in order to formulate the strain mode shape, eigenmode shape and multimode electromechanical FRFs. Unlike analytical techniques, the proposed new numerical solution techniques provided the benefit to analyse the structure with different scalabilities including MEMS devices.

- The comparisons between the numerical solution techniques and experimental studies have been discussed in terms of the variable load resistance, giving good agreement.

Overall, the experimental verification and parametric numerical case studies have been explored and have shown close agreement. For this paper, the proposed new electromechanical finite element modelling has proved that the velocity, current and power FRFs have shown very similar trends with 
the previous published analytical literatures. These numerical results were also validated with experimental studies. Moreover, since the trend of optimal power harvesting FRF using different cases of the tip mass was presented, the new method showed that neglecting the rotary inertia of the tip mass resulted in the resonance frequency error. Notably modelling errors occurred from ignoring the tip mass offset and ignoring both tip mass offset and rotary inertia. The other new aspect shown in using these numerical techniques is that the system models configured normalised strain node and eigenmode shapes using discretised elements for convergence studies. Further detail of frequency analysis using strain node effect in terms of the electrical circuit connection patterns will be a focus of future studies.

The new numerical techniques can be a very useful tool for analysing FRFs, displacement mode shape, and strain node forms. These techniques can be applied for modelling different geometrical aspects for laminated structures and MEMS power harvester devices. For this case, since our main concern is to present the new contribution of numerical techniques, it is important to validate the results obtained from numerical FRFs using experimental studies where the trends of the results were also shown to be very similar with established facts from the previous analytical literatures. Further applications for new aspects of power harvesting using the proposed numerical techniques can be demonstrated in future research studies.

\section{Appendix A. Determining the asymmetric neutral axis}

In figure $\mathrm{A} 1$, the location of the asymmetric neutral axis measured from the $y$-axis to the top surface of the piezoelectric layer can be determined using the resultant force balance in the cross-section of the unimorph structure to give,

$$
\sum_{i=1} F^{(i)}=\int_{-h_{s}+\left(z_{n}-h_{p}\right)}^{z_{n}-h_{p}} \int_{0}^{b} c_{11}^{(1)} z \mathrm{~d} y \mathrm{~d} z+\int_{z_{n}-h_{p}}^{z_{n}} \int_{0}^{b} c_{11}^{(2)} z \mathrm{~d} y \mathrm{~d} z=0
$$

Manipulating equation (A1), the location of the asymmetric neutral axis can be reduced as,

$$
z_{n}=\frac{c_{11}^{(1)} h_{s}{ }^{2}+c_{11}^{(2)} h_{p}{ }^{2}+2 c_{11}^{(1)} h_{s} h_{p}}{2\left(c_{11}^{(1)} h_{s}+c_{11}^{(2)} h_{p}\right)}
$$

where coefficients $\bar{c}_{11}^{(1)}$ and $\bar{c}_{11}^{E}=\bar{c}_{11}^{(2)}$ represent substructure elastic stiffness and piezoelectric elastic stiffness at constant electric field, respectively. 


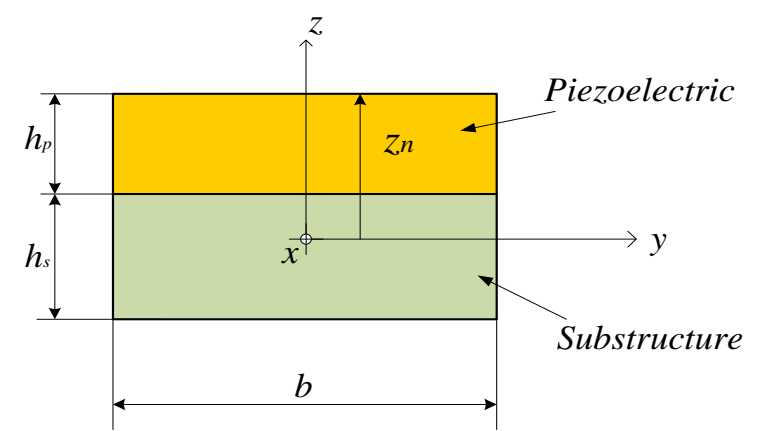

Figure A1. Cross-section of piezoelectric unimorph beam.

\section{Appendix B. Determining the kinetic energy reduced from the kinematic equations}

Corresponding to equations (7) and (11), the total kinetic energy of the system consisting of the elemental unimorph and tip mass offset as shown in figure (3) can be formulated as,

where

$$
K E=K E^{\text {unimorph }}+K E^{\text {tip }}
$$

$$
\begin{gathered}
K E^{\text {unimorph }}=\frac{1}{2} \int_{m^{(1)}} \dot{\boldsymbol{R}}^{\boldsymbol{p} \boldsymbol{p}^{\prime \prime}}(x, z, t) \cdot \dot{\boldsymbol{R}}^{\boldsymbol{p} \boldsymbol{p}^{\prime \prime}}(x, z, t) \mathrm{d} m^{(1)}+\frac{1}{2} \int_{m^{(2)}} \dot{\boldsymbol{R}}^{p \boldsymbol{p}^{\prime \prime}}(x, z, t) \cdot \dot{\boldsymbol{R}}^{p \boldsymbol{p}^{\prime \prime}}(x, z, t) \mathrm{d} m^{(2)} \\
K E^{t i p}=\frac{1}{2} \int_{m^{t i p}} \dot{\boldsymbol{R}}^{m m^{\prime \prime}}\left(x_{\mathrm{e}+1}, z, t\right) \cdot \dot{\boldsymbol{R}}^{m m^{\prime \prime}}\left(x_{\mathrm{e}+1}, z, t\right) \mathrm{d} m^{t i p}
\end{gathered}
$$

As mentioned previously, the translation and angular velocities of tip mass at the end of the unimorph beam length $L$ as shown in figure (1) can be transformed into the local position of the end of the element $x_{e+1}$ as shown in figure (3). Equation (B.2a) can be expanded as,

$$
\begin{aligned}
& K E^{\text {unimorph }}=\frac{1}{2} \int_{m^{(1)}}\left(\dot{\boldsymbol{w}}_{\text {base }}(t) \cdot \dot{\boldsymbol{w}}_{\text {base }}(t)+2 \dot{\boldsymbol{w}}_{\text {base }}(t) \cdot \dot{\boldsymbol{w}}(x, t)+z^{2} \dot{\boldsymbol{\theta}}(x, t) \cdot \dot{\boldsymbol{\theta}}(x, t)+\dot{\boldsymbol{w}}(x, t) \cdot \dot{\boldsymbol{w}}(x, t)\right) \mathrm{d} m^{(1)} \\
& \quad+\frac{1}{2} \int_{m^{(2)}}\left(\dot{\boldsymbol{w}}_{\text {base }}(t) \cdot \dot{\boldsymbol{w}}_{\text {base }}(t)+2 \dot{\boldsymbol{w}}_{\text {base }}(t) \cdot \dot{\boldsymbol{w}}(x, t)+z^{2} \dot{\boldsymbol{\theta}}(x, t) \cdot \dot{\boldsymbol{\theta}}(x, t)+\dot{\boldsymbol{w}}(x, t) \cdot \dot{\boldsymbol{w}}(x, t)\right) \mathrm{d} m^{(2)}
\end{aligned}
$$

It is noted that the first terms for each parenthesis from equation (B.3) must be zero due to the Lagrangian functional form of the system only depending upon the relative translation and velocity of the unimorph as expressed in Section 4.2. The second terms for each parenthesis contribute to the input dynamic forces and these terms can be moved into the non-conservative work section as given in detail in appendix C. For the tip mass kinetic energy, equation (B.2b) can be expanded as,

$$
\begin{aligned}
& K E^{t i p}=\frac{1}{2} \int_{m^{t i p}}\left(\dot{\boldsymbol{w}}_{\text {base }}(t) \cdot \dot{\boldsymbol{w}}_{\text {base }}(t)+2 x_{m} \dot{\boldsymbol{\theta}}\left(x_{e+1}, t\right) \cdot \dot{\boldsymbol{w}}_{\text {base }}(t)+2 x_{m} \dot{\boldsymbol{\theta}}\left(x_{e+1}, t\right) \cdot \dot{\boldsymbol{w}}\left(x_{e+1}, t\right)\right. \\
& +2 x_{c} x_{m} \dot{\boldsymbol{\theta}}\left(x_{e+1}, t\right) \cdot \dot{\boldsymbol{\theta}}\left(x_{e+1}, t\right)+2 z_{c} z_{m} \dot{\boldsymbol{\theta}}\left(x_{e+1}, t\right) \cdot \dot{\boldsymbol{\theta}}\left(x_{e+1}, t\right)+2 x_{c} \dot{\boldsymbol{w}}_{\text {base }}(t) \cdot \dot{\boldsymbol{\theta}}\left(x_{e+1}, t\right) \\
& +2 \dot{\boldsymbol{w}}_{\text {base }}(t) \cdot \dot{\boldsymbol{w}}\left(x_{e+1}, t\right)+\dot{\boldsymbol{w}}\left(x_{e+1}, t\right) \dot{\boldsymbol{w}}\left(x_{e+1}, t\right)+2 x_{c} \dot{\boldsymbol{\theta}}\left(x_{e+1}, t\right) \cdot \dot{\boldsymbol{w}}\left(x_{e+1}, t\right)+x_{c}{ }^{2} \dot{\boldsymbol{\theta}}\left(x_{e+1}, t\right) \cdot \dot{\boldsymbol{\theta}}\left(x_{e+1}, t\right)
\end{aligned}
$$




$$
\left.+z_{c}^{2} \dot{\boldsymbol{\theta}}\left(x_{e+1}, t\right) \cdot \dot{\boldsymbol{\theta}}\left(x_{e+1}, t\right)+z_{m}^{2} \dot{\boldsymbol{\theta}}\left(x_{e+1}, t\right) \cdot \dot{\boldsymbol{\theta}}\left(x_{e+1}, t\right)+x_{m}{ }^{2} \dot{\boldsymbol{\theta}}\left(x_{e+1}, t\right) \cdot \dot{\boldsymbol{\theta}}\left(x_{e+1}, t\right)\right) \mathrm{d} m^{t i p}
$$

Again, the first term of equation (B.4) must be zero. Moreover, the second to fifth terms must be zero due to containing the first mass moment of inertias for the tip mass body about the centroid at point $g$ as shown in figure 3, since the centroid of the tip mass is located on that centroid itself [57]. Again the sixth and seventh terms can be moved into the non-conservative work section.

Equations (B.3)-(B.4) can simply be rewritten in matrix form as,

$$
\begin{array}{r}
K E^{\text {unimorph }}= \\
=\frac{1}{2} \int_{x_{e}}^{x_{e+1}} \int_{A^{(1)}} z^{2} \rho^{(1)} \dot{\boldsymbol{\theta}}(x, t)^{T} \dot{\boldsymbol{\theta}}(x, t) \mathrm{d} A^{(1)} \mathrm{d} x+\frac{1}{2} \int_{x_{e}}^{x_{e+1}} \int_{A^{(2)}} z^{2} \rho^{(2)} \dot{\boldsymbol{\theta}}(x, t)^{T} \dot{\boldsymbol{\theta}}(x, t) \mathrm{d} A^{(2)} \mathrm{d} x \\
+\frac{1}{2} \int_{x_{e}}^{x_{e+1}} \int_{A^{(1)}} \rho^{(1)} \dot{\boldsymbol{w}}(x, t)^{T} \dot{\boldsymbol{w}}(x, t) \mathrm{d} A^{(1)} \mathrm{d} x+\frac{1}{2} \int_{x_{e}}^{x_{e+1}} \int_{A^{(2)}} \rho^{(2)} \dot{\boldsymbol{w}}(x, t)^{T} \dot{\boldsymbol{w}}(x, t) \mathrm{d} A^{(2)} \mathrm{d} x \\
K E^{t i p}=I_{0}^{t i p} x_{c} \boldsymbol{w}\left(x_{e+1}, t\right)^{T} \dot{\boldsymbol{\theta}}\left(x_{e+1}, t\right)+\frac{1}{2} I_{0}^{t i p} \dot{\boldsymbol{w}}\left(x_{e+1}, t\right)^{T} \dot{\boldsymbol{w}}\left(x_{e+1}, t\right)+\frac{1}{2} I_{2}^{t i p} \dot{\boldsymbol{\theta}}\left(x_{e+1}, t\right)^{T} \dot{\boldsymbol{\theta}}\left(x_{e+1}, t\right)
\end{array}
$$

The zeroth and second mass moment of inertias of the tip mass offset can be stated as,

$$
I_{0}^{t i p}=\int_{V^{t i p}} \rho^{t i p} \mathrm{~d} V^{t i p}, \quad I_{2}^{t i p}=\int_{V^{t i p}} \rho^{t i p}\left(z_{m}{ }^{2}+x_{m}{ }^{2}\right) d V^{t i p}+\left(z_{c}{ }^{2}+x_{c}{ }^{2}\right) m^{t i p}=I_{c g}^{t i p}+m^{t i p}\left(z_{c}{ }^{2}+x_{c}{ }^{2}\right)
$$

where $\mathrm{d} V^{t i p}=\mathrm{d} x_{m} \mathrm{~d} y_{m} \mathrm{~d} z_{m}$ and definite integral forms of $f(y, z)$ implied from equation (B.5) can be defined as,

$$
\int_{A^{(1)}} f(y, z) \mathrm{d} A^{(1)}=\int_{-h_{s}+\left(z_{n}-h_{p}\right)}^{z_{n}-h_{p}} \int_{0}^{b} f(y, z) \mathrm{d} y \mathrm{~d} z, \int_{A^{(2)}} f(y, z) \mathrm{d} A^{(2)}=\int_{z_{n}-h_{p}}^{z_{n}} \int_{0}^{b} f(y, z) \mathrm{d} y \mathrm{~d} z, f(y, z) \in\left\{1, z, z^{2}\right\}
$$

Note that equation (B.7) can be used for any operational integrations not only in the kinetic energy derivation but also for other Lagrangian energy forms given from equations (36)-(39).

\section{Appendix C. Determining the non-conservative work from the input base motion}

As mentioned in appendix $\mathrm{B}$, the contributions of non-conservative forces due to the input base excitation on the system were reduced from the kinetic energy. As a result, the virtual work done by non-conservative forces can simply be formulated in terms of their time dependent response using the Hamiltonian principle as,

$$
\delta W F^{\text {base }}=\int_{t_{1}}^{t_{2}} \boldsymbol{F} . \delta \boldsymbol{r} \mathrm{d} t
$$

Note that from the mathematical standpoint, the functional form of Lagrangian's principle is reduced from the Hamiltonian's principle using the variational method. Therefore, corresponding to equation 
(39), the virtual non-conservative works can be expanded by using the second terms for each parenthesis from equation (B.3) and the sixth and seventh terms from equation (B.4) as,

$$
\begin{aligned}
\delta W F^{\text {base }} & =\frac{1}{2}\left(\int_{t_{1} m^{(1)}}^{t_{2}} 2 \dot{\boldsymbol{w}}_{\text {base }}(t) \cdot \delta \dot{\boldsymbol{w}}(x, t) \mathrm{d} m^{(1)} \mathrm{d} t+\int_{t_{1} m^{(2)}}^{t_{2}} 2 \dot{\boldsymbol{w}}_{\text {base }}(t) \cdot \delta \dot{\boldsymbol{w}}(x, t) \mathrm{d} m^{(2)} \mathrm{d} t\right. \\
& \left.+\int_{t_{1}}^{t_{2}} \int_{m^{t i p}} 2 x_{c} \dot{\boldsymbol{w}}_{\text {base }}(t) \cdot \delta \dot{\boldsymbol{\theta}}\left(x_{e+1}, t\right) \mathrm{d} m^{t i p} \mathrm{~d} t+\int_{t_{1} m^{t i p}}^{t_{2}} \int_{\dot{\boldsymbol{w}}_{\text {base }}}(t) \cdot \delta \dot{\boldsymbol{w}}\left(x_{e+1}, t\right) \mathrm{d} m^{t i p} \mathrm{~d} t\right)
\end{aligned}
$$

Equation (C.2) needs to be further formulated by applying partial integration and the result can be stated in the matrix form as,

$$
\begin{aligned}
\delta W F^{\text {base }}= & -\int_{t_{1}}^{t_{2}} \int_{x_{e}}^{x_{e+1}} \int_{A^{(1)}} \rho^{(1)} \ddot{\boldsymbol{w}}_{\text {base }}(t) \delta \boldsymbol{w}(x, t)^{T} \mathrm{~d} A^{(1)} \mathrm{d} x \mathrm{~d} t-\int_{t_{1}}^{t_{2}} \int_{x_{e}}^{x_{e+1}} \int_{A^{(2)}} \rho^{(2)} \ddot{\boldsymbol{w}}_{\text {base }}(t) \delta \boldsymbol{w}(x, t)^{T} \mathrm{~d} A^{(2)} \mathrm{d} x \mathrm{~d} t \\
& -\int_{t_{1}}^{t_{2}} I_{0}^{t i p} x_{c} \ddot{\boldsymbol{w}}_{\text {base }}(t) \delta \boldsymbol{\theta}\left(x_{e+1}, t\right)^{T} \mathrm{~d} t-\int_{t_{1}}^{t_{2}} I_{0}^{t i p} \ddot{\boldsymbol{w}}_{\text {base }}(t) \delta \boldsymbol{w}\left(x_{e+1}, t\right)^{T} \mathrm{~d} t
\end{aligned}
$$

\section{Appendix D. Determining geometry parameters of tip mass offset}

Corresponding to the parameters given from equation (B.6), the mass moment of inertias of the tip mass offset as shown in figure 4 can be formulated. Note that as mentioned previously, the extra unimorph beam length as shown in figure D1 also contributed to the tip mass offset. In this case, the zeroth mass moment of inertia can be stated as,

$$
I_{0}^{t i p}=\rho^{t i p} b l_{t} h_{t}+\rho^{(1)} b l_{t} h_{s}
$$

and the second mass moment of inertia of tip mass offset at the point $d$ located in the end of unimorph beam can be reduced to give,

$$
\begin{aligned}
& I_{2}^{t i p}=\rho^{t i p} b l_{t} h_{t}\left(\frac{\left(l_{t}^{2}+h_{t}^{2}\right)}{12}+\left(\frac{h_{t}}{2}-h_{p}-\bar{z}_{c}+z_{n}\right)^{2}\right) \\
& +\rho^{(1)} b l_{t} h_{s}\left(\frac{\left(l_{t}^{2}+h_{s}^{2}\right)}{12}+\left(\frac{h_{s}}{2}+h_{p}-z_{n}+\bar{z}_{c}\right)^{2}\right)+I_{O}^{t i p}\left(\bar{z}_{c}{ }^{2}+\bar{x}_{c}{ }^{2}\right)
\end{aligned}
$$

where the offset distances measured from the tip mass centroid to the point $d$ in the x-and $z$-axes can respectively be formulated as,

$$
\begin{gathered}
\bar{x}_{c}=\frac{\rho^{t i p} b l_{t}{ }^{2} h_{t}+\rho^{(1)} b l_{t}{ }^{2} h_{s}}{2\left(\rho^{t i p} b l_{t} h_{t}+\rho^{(1)} b l_{t} h_{s}\right)} \\
\bar{z}_{c}=\frac{\rho^{t i p} b l_{t} h_{t}\left(\frac{h_{t}}{2}+z_{n}-h_{p}\right)+\rho^{(1)} b l_{t}\left(z_{n}-h_{p}\right)\left(\frac{z_{n}-h_{p}}{2}\right)-\rho^{(1)} b l_{t}\left(h_{s}+h_{p}-z_{n}\right)\left(\frac{h_{s}+h_{p}-z_{n}}{2}\right)}{\rho^{t i p} b l_{t} h_{t}+\rho^{(1)} b l_{t}\left(z_{n}-h_{p}\right)+\rho^{(1)} b l_{t}\left(h_{s}+h_{p}-z_{n}\right)}
\end{gathered}
$$


Alternatively with the same result, the second mass moment of inertia of tip mass offset at the point $d$ located at the end of the unimorph beam can straightforwardly be formulated as,

$$
\begin{aligned}
& I_{2}^{t i p}=\rho^{t i p} b l_{t} h_{t}\left(\frac{\left(l_{t}^{2}+h_{t}^{2}\right)}{12}+\left(z_{n}-h_{p}+\frac{h_{t}}{2}\right)^{2}+\left(\frac{l_{t}}{2}\right)^{2}\right) \\
& +\rho^{(1)} b l_{t} h_{s}\left(\frac{\left(l_{t}^{2}+h_{s}^{2}\right)}{12}+\left(\frac{l_{t}}{2}\right)^{2}+\left(\frac{h_{s}}{2}-\left(z_{n}-h_{p}\right)\right)^{2}\right)
\end{aligned}
$$

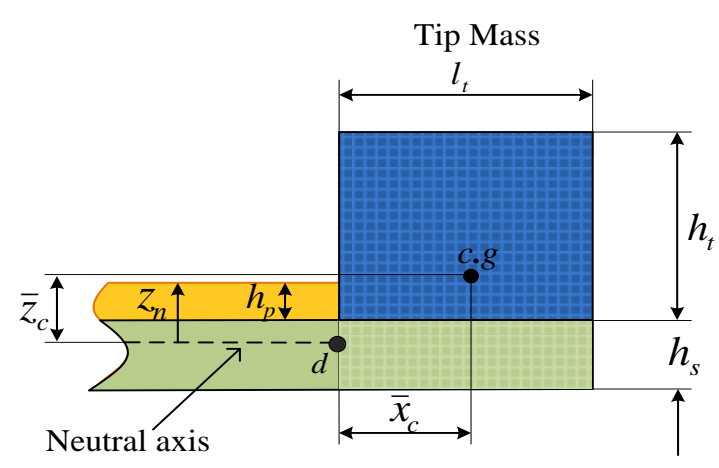

Figure D1. Geometry of tip mass offset.

\section{References}

[1] Zuo L and Tang X 2013 Large-scale vibration energy harvesting J. Intell. Mater. Syst. Struct. 24 1405-30

[2] Sue C-Y and Tsai N-C 2012 Human powered MEMS-based energy harvest devices App Energy 93 390-403

[3] Kim H U, Lee W H, Dias H V R and Priya S 2009 Piezoelectric microgenerators-current status and challenges IEEE Trans. Ultrason. Ferroelectr. Freq. Control 56 1555-68

[4] Priya S, Ryu J, Park C-S, Oliver J, Choi J-J and Park D-S 2009 Piezoelectric and magnetoelectric thick films for fabricating power sources in wireless sensor nodes Sensors 9 $6362-84$

[5] Hande A, Bridgelall R and Zoghi B 2010 Vibration energy harvesting for disaster asset monitoring using active RFID tags Proc. of the IEEE 9 1620-28

[6] Chao P C-P 2011 Energy harvesting electronics for vibratory devices in self-powered sensors IEEE Sensor J. 11 3106-21

[7] Kim H S, Kim J-H and Kim J 2011 A review of piezoelectric energy harvesting based on vibration Int. J. Prec. Eng. Manufact. 12 1129-41

[8] Roundy S and Wright P K 2004 A piezoelectric vibration based generator for wireless electronics Smart Mater. Struct. 18 1131-42

[9] Takeda H, Mihara K, Yoshimura T, Hoshina T and Tsurumi T 2011 Effect of material constants on power output in piezoelectric vibration-based generators IEEE Trans. Ultrason. Ferroelectr. Freq. Control 58 1852-59 
[10] Liang J and Liao W-H 2012 Impedance modeling and analysis for piezoelectric energy harvesting Systems IEEE/ASME Trans. Mechatronics 17 1145-57

[11] duToit N E, Wardle B L and Kim S 2005 Design considerations for MEMS scale piezoelectric mechanical vibration energy harvesters Int. J. Integr. Ferroelectr. 71 121-160

[12] Liao Y and Sodano H 2008 Model of a single mode energy harvester and properties for optimal power generation Smart Mater. Struct. 17065026

[13] Goldschmidtboeing F and Woias P 2008 Characterization of different beam shapes for piezoelectric energy harvesting J. Micromech. Microeng. 18104013

[14] Kim M, Hoegen M, Dugundji J and Wardle B L 2010 Modeling and experimental verification of proof mass effects on vibration energy harvester performance Smart Mater. Struct. 19 045023

[15] Erturk A 2012 Assumed-modes modeling of piezoelectric energy harvesters: Euler-Bernoulli, Rayleigh, and Timoshenko models with axial deformations Comp. Struct. 106-107 214-227

[16] Erturk A and Inman D J 2009 An experimentally validated bimorph cantilever model for piezoelectric energy harvesting from base excitations Smart Mater. Struct. 18025009

[17] Wickenheiser A M and Garcia E 2010 Broadband vibration-based energy harvesting improvement through frequency up-conversion by magnetic excitation Smart Mater. Struct. 19 065020

[18] Lumentut M F and Howard I M 2013 Analytical and experimental comparisons of electromechanical vibration response of a piezoelectric bimorph beam for power harvesting Mech. Syst. Signal Proc. 36 66-86

[19] Lumentut M F and Howard I M 2011 Analytical modeling of self-powered electromechanical piezoelectric bimorph beams with multidirectional excitation Int. J. Smart Nano Mater. 2 134175

[20] Lumentut M F and Howard I M 2012 Electromechanical piezoelectric power harvester frequency response modelling using closed-form boundary value methods IEEE/ASME Trans. Mechatronics in press http://dx.doi.org/10.1109/TMECH.2012.2219066

[21] Lumentut M F, Francis L A and Howard I M 2012 Analytical techniques for broadband multielectromechanical piezoelectric bimorph beams with multifrequency power harvesting IEEE Trans. Ultrason. Ferroelectr. Freq. Control 59 1555-68

[22] Allik H and Hughes T J R 1970 Finite element method for piezoelectric vibration Int. J. Numer. Methods Engng. 2 151-7

[23] Allik H, Webman K M and Hunt J T 1974 Vibrational response of sonar transducers using piezoelectric finite elements J Acoust Soc Am. 56 1782-91

[24] Kagawa Y and Yamabuchi T 1976 Finite element approach for a piezoelectric circular rod IEEE Trans. Sonics Ultrason. SU-23 379-384 
[25] Naillon M, Coursant R H and Besner F 1983 Analysis of piezoelectric structures by a finite element method ACTA Electronica 25 341-362

[26] Tzou H S and Tzeng C I 1983 Distributed piezoelectric sensor/actuator design for dynamic measurement/control of distributed parameter system: A piezoelectric finite element approach J. Sound Vib. 138 17-34

[27] Saravanos D A, Heyliger P R and Hopkins D A 1996 Layerwise mechanics and finite element for the dynamics analysis of piezoelectric composite plate Int. J. Solids Struct. 34 359-378

[28] Moita J M, Correia I F P and Soares C M M 2004 Active control of adaptive laminated structures with bounded piezoelectric sensors and actuators Comp. Struct. 82 1349-58

[29] Lam K Y, Peng X Q, Liu G R and Reddy J N 1997 A finite-element model for piezoelectric composite laminates Smart Mater. Struct. 6 583-91

[30] Gaudenzi P, Carbonaro R and Benzi E 2000 Control of beam vibrations by means of piezoelectric devices: theory and experiments Compos. Struct. 50 373-9

[31] Detwiler D T, Shen H M-H and Venkayya V B 1995 Finite element analysis of laminated composites tructures containing distributed piezoelectric actuators and sensors Fin. Elem. Anal. Des. 20 87-100

[32] Fernandes A and Pouget J 2003 Analytical and numerical approach to piezoelectric bimorph Int. J. Solids Struct. $404331-52$

[33] Wang S Y 2004 A finite element model for the static and dynamic analysis of a piezoelectric bimorph Int. J. Solids Struct. 41 4075-96

[34] Thomas O, Deü J-F and Ducarne J 2009 Vibrations of an elastic structure with shunted piezoelectric patches: efficient finite element formulation and electromechanical coupling coefficients Int. J. Numer. Methods Engng. 8 235-268

[35] Lumentut M F, Teh K K and Howard I M 2008 Computational FEA model of a coupled piezoelectric sensor and plate structure for energy harvesting Aust. J. Mech. Engng. 5 199-208

[36] DeMarqui Jr. C, Erturk A and Inman D J 2009 An electromechanical finite element model for piezoelectric energy harvester plates J. Sound Vib. 327 9-25

[37] Aladwani A, Arafa M, Aldraihem O and Baz A 2012 Cantilevered piezoelectric energy harvester with a dynamic magnifier ASME J. Vib. Acoust. 134031004

[38] Zhu M, Worthington E and Njuguna J 2009 Analyses of power output of piezoelectric energyharvesting devices directly connected to a load resistor using a coupled piezoelectric-circuit finite element method IEEE Trans. Ultrason. Ferroelectr. Freq. Control 56 1309-18

[39] Yang Y and Tang L 2009 Equivalent circuit modeling of piezoelectric energy harvesters $J$. Intell. Mater. Syst. Struct. 20 2223-35

[40] Tiersten H F 1969 Linear piezoelectric plate vibrations (Plenum Press) 
[41] Nye J F 1984 Physical properties of crystals: their representation by tensors and matrices, (Oxford, England: Clarendon Press)

[42] H. Tanaka, Generalized basic equations for bending motions of piezoelectric bars formulated from Hamilton's principle J. Acoustic. Soc. Am. 4 (1993) 1764-1772.

[43] Standards Committee of the IEEE Ultrasonics, Ferroelectrics, and Frequency Control Society 1987 IEEE standard on piezoelectricity (New York: IEEE)

[44] Lumentut M F 2011 Mathematical dynamics of electromechanical piezoelectric energy harvester $P h D$ thesis Curtin University, Australia (http://espace.library.curtin.edu.au/R/?func=dbin-jumpfull\&object_id=186549\&local_base=gen01-era02)

[45] Jeon Y.B, Sood R, Jeong J-H and Kim S-G 2005 MEMS power generator with transverse mode thin film PZT Sens. Actuators A 122 16-22

[46] Fang H B, Liu J Q, Xu Z Y, Dong L, Wang L, Chen D, Cai B C and Liu Y 2006 Fabrication and performance of MEMS-based piezoelectric power generator for vibration energy harvester Microelectron. J. 37 1280-84

[47] Elfrink R, Kamel T M, Goedbloed M, Matova S, Hohlfeld D, van Andel Y and van Schaijk R 2009 Vibration energy harvesting with aluminum nitride-based piezoelectric devices $J$. Micromech. Microeng. 19094005

[48] Andosca R, McDonald T G, Genova V, Rosenberg S, Keating J, Benedixen C and Wu J 2012 Experimental and theoretical studies on MEMS piezoelectric vibrational energy harvesters with mass loading Sens. Actuators A 178 76-87

[49] Miller L M, Halvorsen E, Dong T and Wright P K 2011 Modeling and experimental verification of low-frequency MEMS energy harvesting from ambient vibrations J. Micromech. Microeng. 21045029

[50] Park J C, Park J Y and Lee Y-P 2010 Modeling and characterization of piezoelectric $d 33$-mode MEMS energy harvester IEEE/ASME J. Microelectromech. Syst. 19 1215-22

[51] Dankert H and Dankert J 1995 Technische mechanik vol. 2 (Stuttgart: B.G. Teubner)

[52] Ikeda T 1990 Fundamentals of piezoelectricity (New York: Oxford University Press)

[53] Zienkiewicz O C and Taylor R L 2005 The finite element method for solid and structural mechanics, Sixth ed. (UK: Butterworth-Heinemann)

[54] Weaver Jr. W W and Johnston P R 1984 Finite elements for structural analysis (United States: Prentice-Hall)

[55] Reddy J N 1984 An introduction to the finite element method (New York: McGraw-Hill)

[56] Meirovitch L 1967 Analytical methods in vibrations (United States: Prentice Hall)

[57] Beer F P and Johnston Jr E R 1977 Vector mechanics for engineers: statics and dynamics (New York: McGraw-Hill) 